\title{
Computational Fluid Dynamic Analysis of the VHTR Lower Plenum Standard Problem
}

Richard W. Johnson

Richard R. Schultz

July 2009
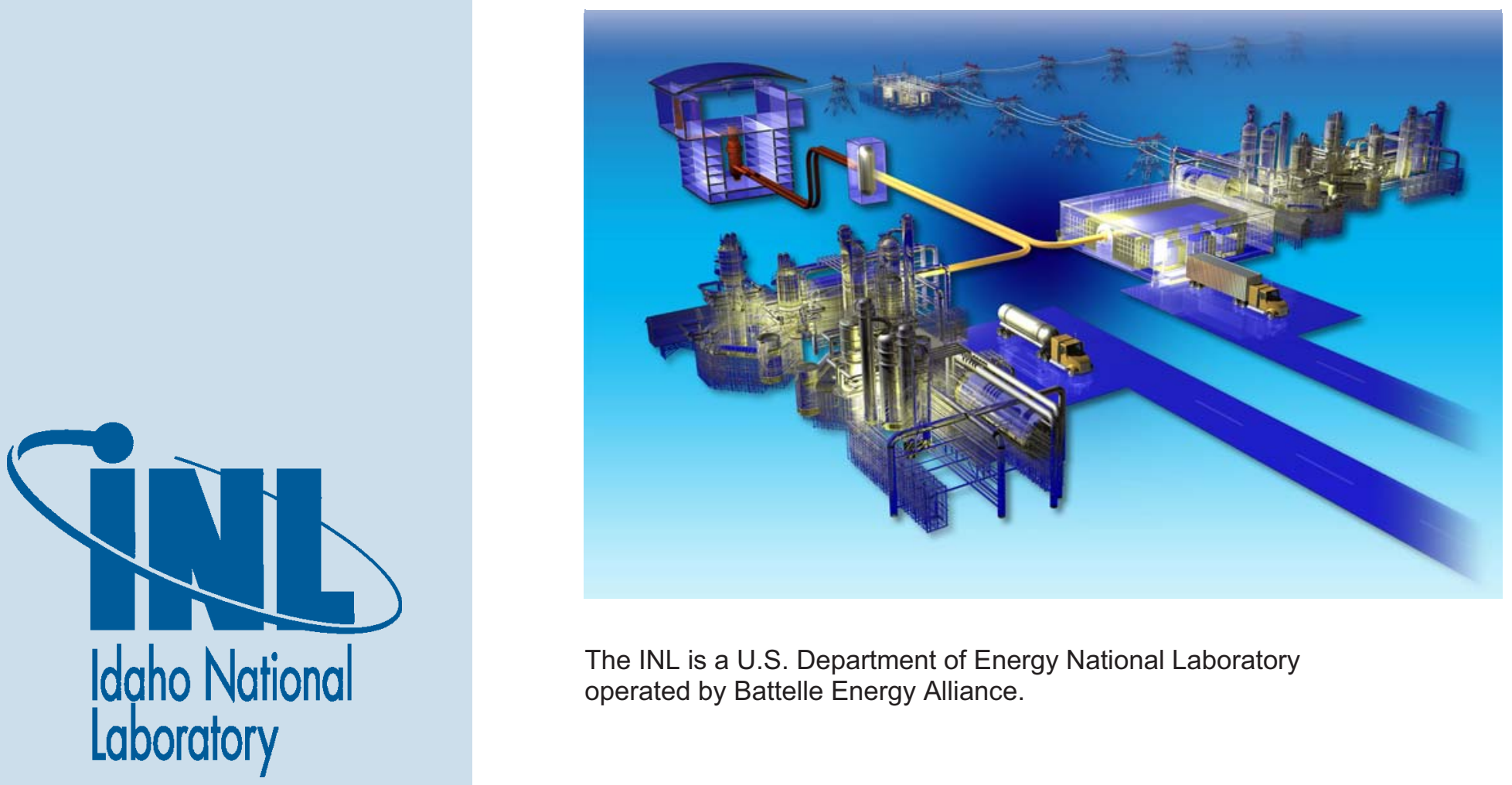

The INL is a U.S. Department of Energy National Laboratory operated by Battelle Energy Alliance. 


\section{DISCLAIMER}

This information was prepared as an account of work sponsored by an agency of the U.S. Government. Neither the U.S. Government nor any agency thereof, nor any of their employees, makes any warranty, expressed or implied, or assumes any legal liability or responsibility for the accuracy, completeness, or usefulness, of any information, apparatus, product, or process disclosed, or represents that its use would not infringe privately owned rights. References herein to any specific commercial product, process, or service by trade name, trade mark, manufacturer, or otherwise, does not necessarily constitute or imply its endorsement, recommendation, or favoring by the U.S. Government or any agency thereof. The views and opinions of authors expressed herein do not necessarily state or reflect those of the U.S. Government or any agency thereof. 


\section{Computational Fluid Dynamic Analysis of the VHTR Lower Plenum Standard Problem}

Richard W. Johnson

Richard R. Schultz

July 2009

Idaho National Laboratory

Next Generation Nuclear Plant Project

Idaho Falls, Idaho 83415

Prepared for the

U.S. Department of Energy

Office of Nuclear Energy

Under DOE Idaho Operations Office

Contract DE-AC07-05ID14517 



\section{Next Generation Nuclear Plant Project}

\section{Computational Fluid Dynamic Analysis of the VHTR Lower Plenum Standard Problem}

INL/EXT-09-16325

July 2009
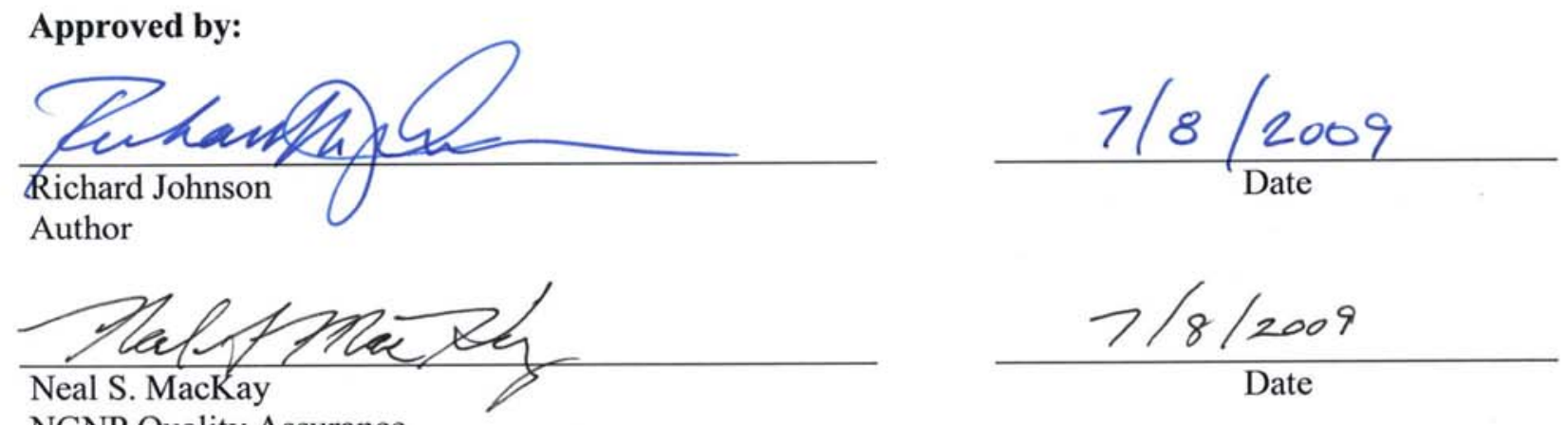

NGNP Quality Assurance
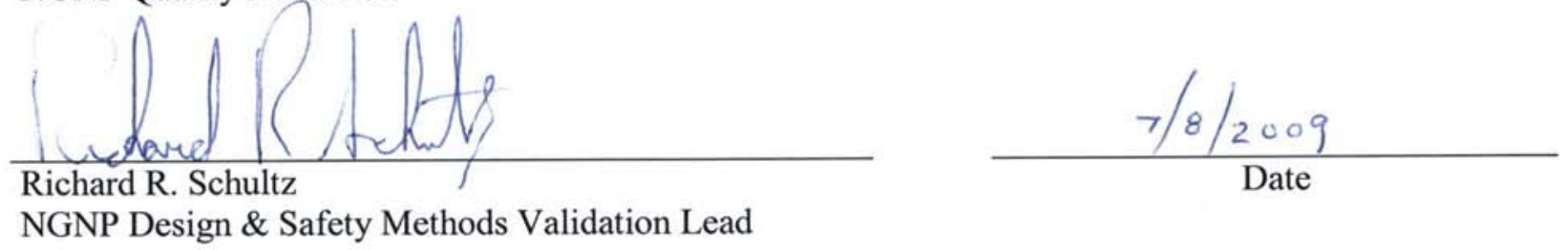



\section{SUMMARY}

The U.S. Department of Energy is promoting the resurgence of nuclear power in the United States for both electrical power generation and production of process heat required for industrial processes such as the manufacture of hydrogen for use as a fuel in automobiles. The project DOE is funding to accomplish this is called the Next Generation Nuclear Plant (NGNP) Project, which is based on a Generation IV reactor concept called the very high temperature reactor (VHTR). The VHTR will use helium as the coolant at temperatures ranging from $450^{\circ} \mathrm{C}$ to perhaps $1000^{\circ} \mathrm{C}$. While computational fluid dynamics (CFD) has not previously been used for the safety analysis of nuclear reactors in the United States, it is being considered for existing and future reactors. It is fully recognized that CFD simulation codes will have to be validated for flow physics reasonably close to actual fluid dynamic conditions expected in normal and accident operational situations. To this end, experimental data have been obtained in a scaled model of a narrow slice of the lower plenum of a prismatic VHTR. This report presents results of CFD examinations of these data to explore potential issues with the geometry, initial conditions, flow dynamics, and data needed to fully specify the inlet and boundary conditions; results for several turbulence models are examined. Issues are addressed and recommendations about the data are made.

Figure S-1 is an isometric view of the scaled model of a slice of the VHTR lower plenum that was installed in INL's matched index of refraction (MIR) facility, showing the inlets, outlet, and the direction of flow. Figure S-2 shows a plan view of the scaled model.

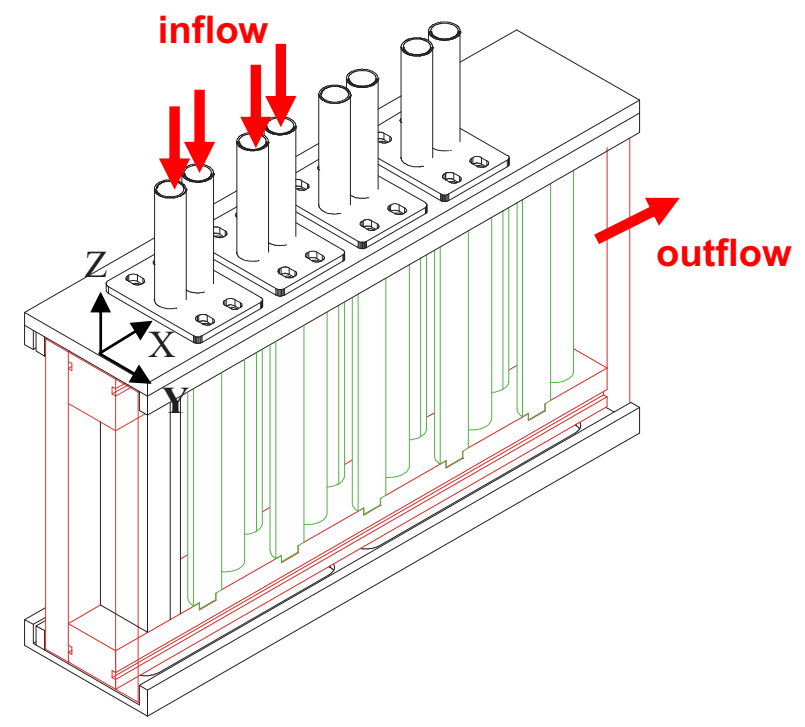

Figure S-1. Isometric view of the scaled model.

Inlet jets (open)

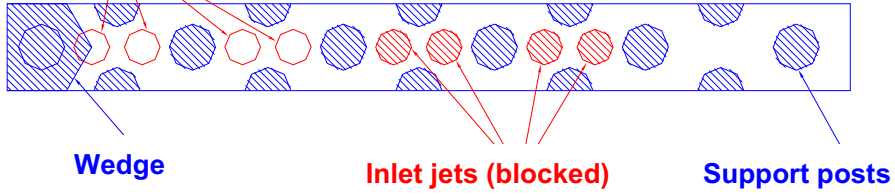

Figure S-2. Plan view of the scaled model. 
Accurate and complete inlet boundary conditions are critical to the usefulness of any CFD validation data set. The inlet mass flows for the MIR facility were measured by mass flow rotameters with each inlet duct having its own rotameter. The inlet mass flows were physically adjusted based on these rotameters. The rotameters provide accurate mass flow rates. During the course of the data taking, particle-image velocimetry (PIV) data were taken for a vertical stretch of the inlet ducts above the jet inlet plane of the scaled model. The data were then processed by the PIV software. Data at $Z=9.7 \mathrm{~mm}$ and $Z=11 \mathrm{~mm}$ (above the inlet plane) were extracted for use as inlet conditions.

Figure S-3 visually compares the mass flow rates for the four jets based on the rotameter flow rates and the PIV data at 9.7 and $11 \mathrm{~mm}$. As can be seen, the mass flow rates for the PIV data are all lower than those from the rotameters. The vertical velocity data points for the $11 \mathrm{~mm}$ PIV data were all multiplied by one factor to yield the same total inlet mass flow rate as for the uniform inlet case. The flow rates for these modified $11 \mathrm{~mm}$ data are also shown in Figure S-2. The discrepancy between the rotameter flow data and the PIV inlet data is a serious shortcoming of the MIR data and should be remedied. It is recommended that additional MIR data be taken and that the inlet ducts be measured with laser sheets that illuminate the duct cross-sections such that dense inlet data can be obtained that should provide close agreement with the mass flow rotameters.

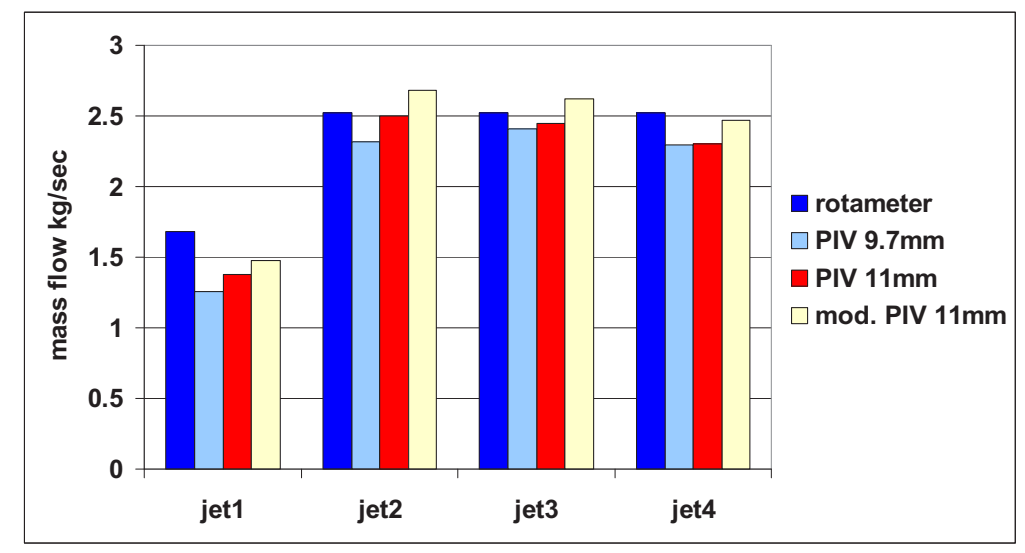

Figure S-3. Comparison of mass flow rates for the four inlet jets derived from rotameter data and detailed PIV measurements at $Z=9.7$ and $11 \mathrm{~mm}$ and the modified PIV data at $11 \mathrm{~mm}$.

The scaled model shown in Figure S-1, which is $104.8 \mathrm{~mm}$ wide, was positioned inside the test section of the INL's MIR facility. The cross section of the MIR test section is $609.6 \mathrm{~mm}$ square. The mineral oil working fluid not only flows into the scaled model, but around the outside of the model as well. Consequently, the oil from the outer flow joins the oil from the inner flow at the model exit. A concern regarding the flow in the scaled model is that the outer flow alters the flow at the exit of the scaled model, possibly affecting the flow inside the model. A two-dimensional study was performed to assess the affects of the outer flow on the inner flow to determine whether the outer flow would need to be included in the CFD model. The study found that only the interior flow needs to be computed if a constant pressure outlet boundary condition is employed.

The flow in the scaled model is turbulent and a turbulence model must be used to represent the effects of turbulence. Four turbulence models are employed, including three eddy-viscosity models and the sophisticated Reynolds stress model. Figure S-4 provides contour plots of vertical velocity contours for the four turbulence models and the MIR data at a horizontal plane that is about one-third the way down from the inlet plane of the scaled model. The standard $k \sim \varepsilon$ model, Figure S-4a, and the Reynolds stress model, Figure S-4d, show skewed asymmetric signatures for the first two jets, located at the left end. The Abe, Kondoh, and Nagano (AKN) $k \sim \varepsilon$ model, Figure S-4b, and the Menter $k \sim \omega$ model, Figure S-4c, on the other hand, show symmetric jet signatures as do the MIR data, Figure S-4e. 


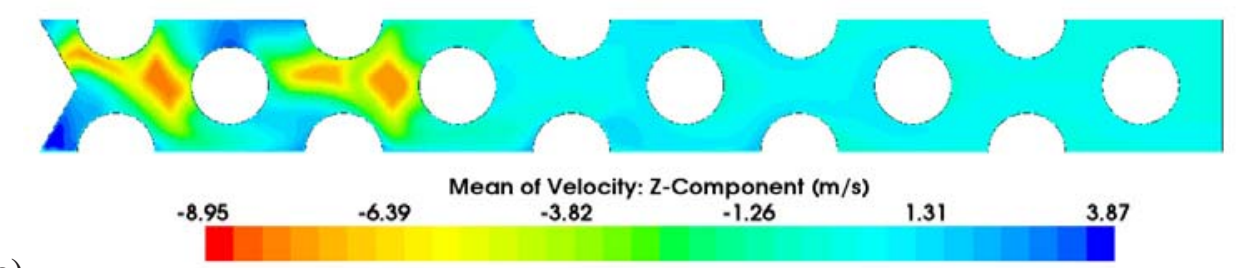

(a)

(b)
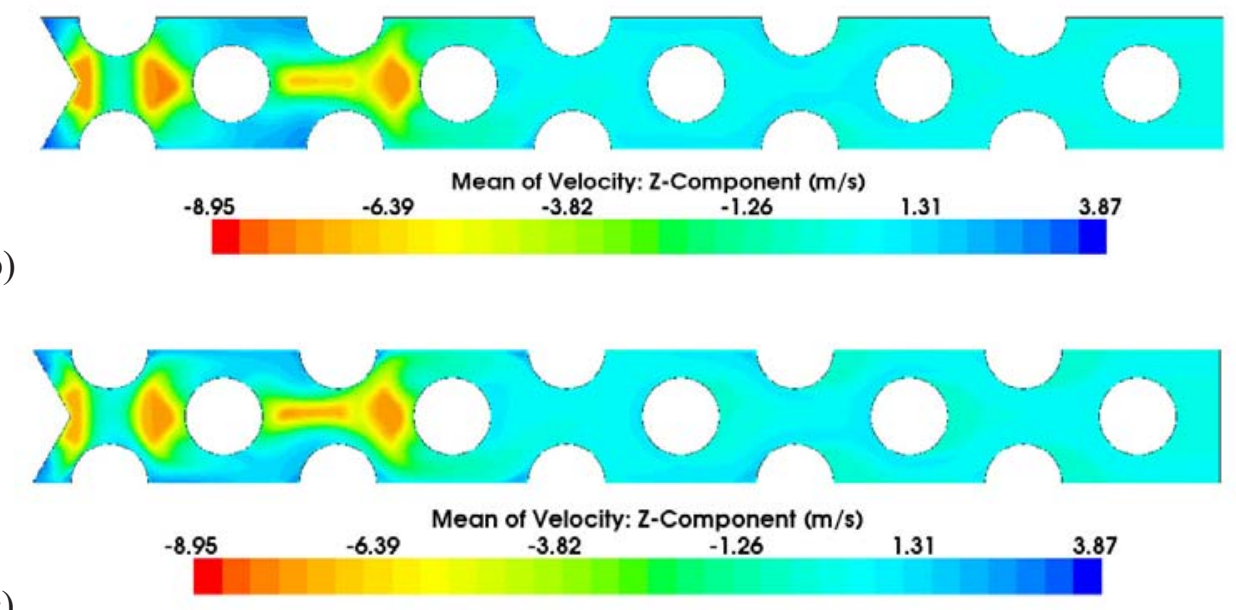

(c)

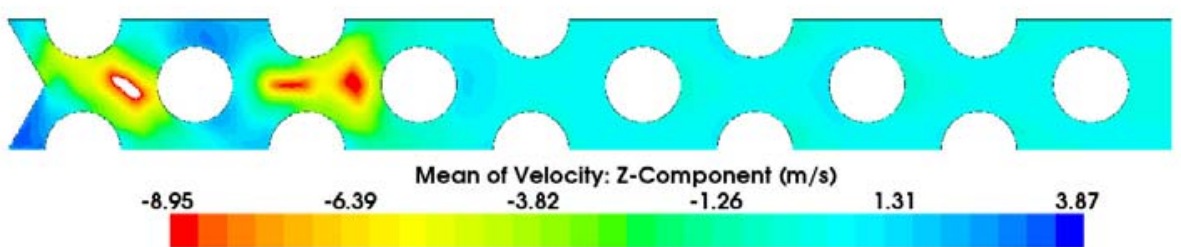

(d)

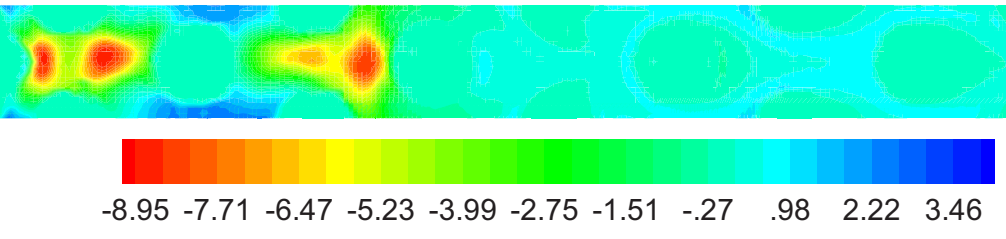

Figure S-4. Contour plots for time mean vertical velocity $W$ at $\mathrm{Z}=-70 \mathrm{~mm}$ for the (a) standard $k \sim \varepsilon$ model, (b) AKN model, (c) Menter $k \sim \omega$ model, Reynolds stress model, and (e) MIR data.

The results for the Reynolds stress model, Figure S-4d, fail to show a signature for the first jet (at the left). This is because the inlet data for this case are taken from the PIV data (modified $11 \mathrm{~mm}$ ), which are too low. The other results shown in Figure S-4 all use the inlet data based on the mass flow rotameters. Additionally, results for the Reynolds stress model progress to either steady flow or to slowly oscillating flow with small oscillation amplitude, while results for the AKN and $k \sim \omega$ model show very dynamic flow patterns. Figure S-5 plots time signatures of nonstationary velocity at several points in the flow for the Reynolds stress model and the AKN model. 

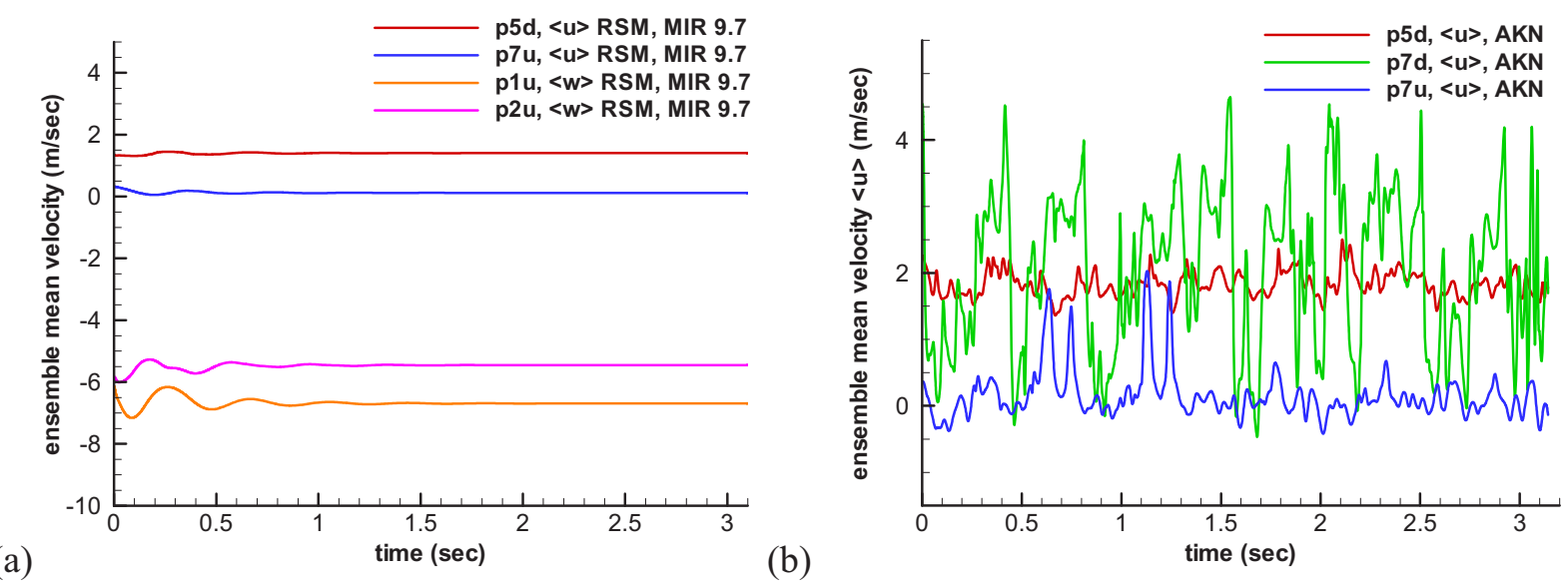

Figure S-5. Time signatures at various points for the (a) Reynolds stress model and (b) AKN model.

The MIR data were taken at a very slow frequency (2-3 per second), which is not rapid enough to capture the vortex shedding (about 35 times per second). It is recommended that new data be taken with much faster data acquisition to be able to better characterize the true flow phenomena and help sort out which turbulence models are giving the most accurate results.

Finally, it is clear from the above CFD investigation that CFD should be used in the design of new standard problems. The application of CFD will help the experiment to be designed to have inlet and outlet boundaries that can be properly simulated and will help in characterizing the flow itself to know what measurements are best, at which locations, using which data acquisition techniques. CFD will also help suggest which phenomena should be examined separately in simpler standard problems, primarily to see how well various turbulence models perform. 


\section{CONTENTS}

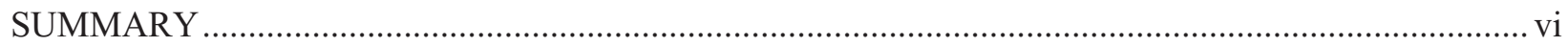

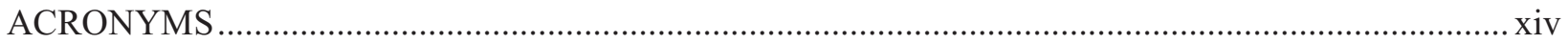

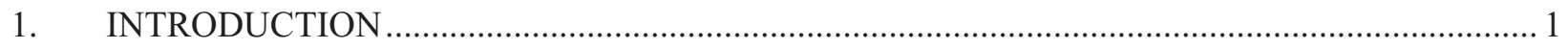

2. NONSTATIONARY TURBULENT FLUID DYNAMICS …................................................ 3

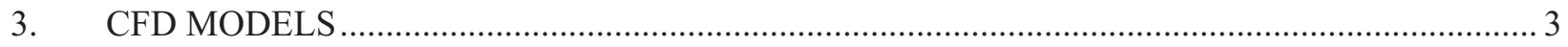

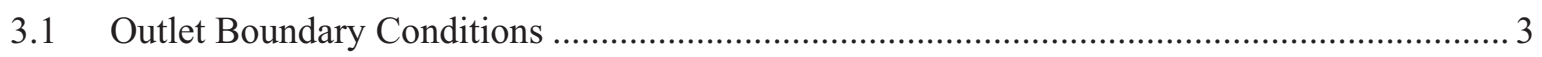

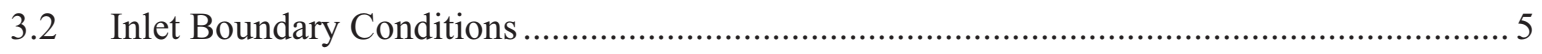

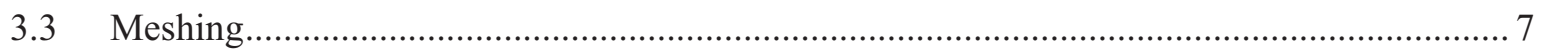

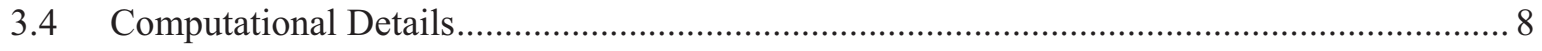

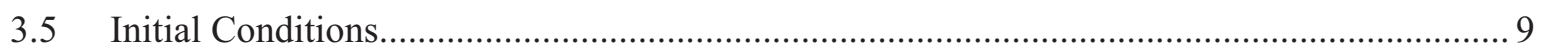

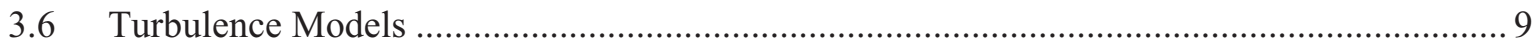

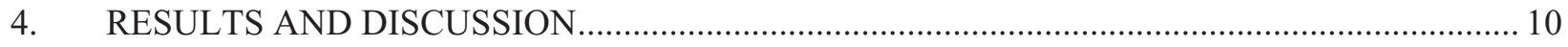

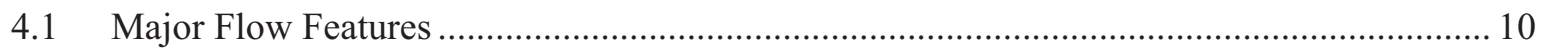

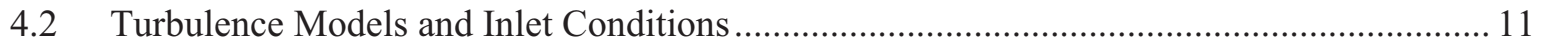

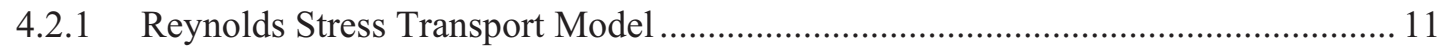

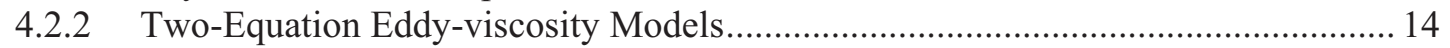

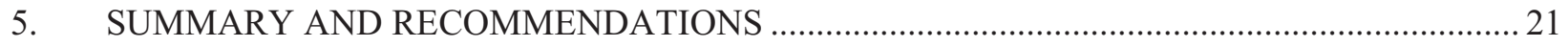

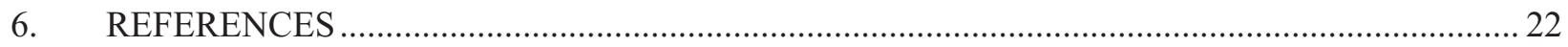

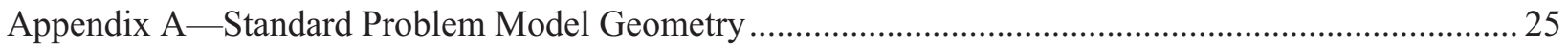

\section{FIGURES}

Figure S-1. Isometric view of the scaled model.....................................................................................

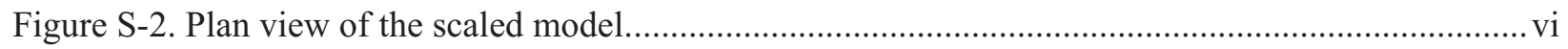

Figure S-3. Comparison of mass flow rates for the four inlet jets derived from rotameter data and detailed PIV measurements at $Z=9.7$ and $11 \mathrm{~mm}$ and the modified PIV data at $11 \mathrm{~mm}$.........vii

Figure S-4. Contour plots for time mean vertical velocity $W$ at $\mathrm{Z}=-70 \mathrm{~mm}$ for the (a) standard $k \sim \varepsilon$ model, (b) AKN model, (c) Menter $k \sim \omega$ model, Reynolds stress model, and (e) MIR data.

Figure S-5. Time signatures at various points for the (a) Reynolds stress model and (b) AKN

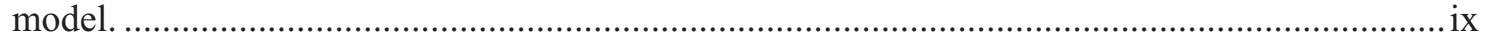

Figure 1. Plan view of the VHTR lower plenum. ....................................................................... 1

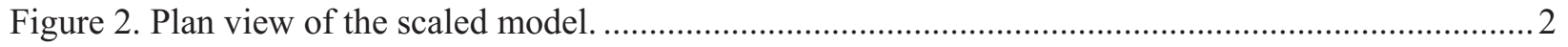

Figure 3. Isometric view of the scaled model and origin and coordinate axes for the CFD model.............2 
Figure 4. Stream function contours of the flow in the CFD model, which includes the outer flow showing vortex-shedding behind the physical model in the MIR test loop.

Figure 5. Profiles of the time mean velocity $U$ at the (a) outlet plane of the scaled model and (b) profile location $3 \mathrm{f}$ (see Figure 4), for cases using pressure-outlet and outflow boundary conditions compared to the reference inner-outer case.

Figure 6. Profiles of the (a) time mean velocity $V$ at profile location $3 \mathrm{f}$ (see Figure 4 ) and (b) Reynolds stress $\bar{u} \bar{v}$ at $3 \mathrm{f}$ for cases using pressure-outlet and outflow boundary conditions compared to the reference inner-outer case.

Figure 7. Comparison of mass flow rates for the four inlet jets derived from rotameter data and detailed PIV measurements at $Z=9.7$ and $11 \mathrm{~mm}$ and the modified PIV data at $11 \mathrm{~mm}$.

Figure 8 . A view of the bottom plane of the a3 grid and an isometric view of the same grid showing inlet ports (green), outlet plane (red), and walls (gray)...... 7

Figure 9. Locations of profiles and points for data comparison. ........................................................ 8

Figure 10. Time mean velocity $W$ along data profile ' $\mathrm{y} 0 \mathrm{u}$ ' for five different time intervals..................... 8

Figure 11. Comparisons of results for the AKN turbulence model starting from a semiconverged steady initial condition and from a stagnant condition to results for the RSM from stagnant initial conditions and the MIR data for (a) W at y0u, (b) W at y0d, (c) $U$ at $\mathrm{x} 4 \mathrm{~d}$, and (d) $\mathrm{U}$ at $\mathrm{x} 6 \mathrm{~d}$

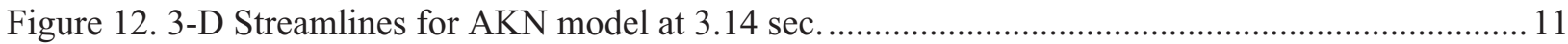

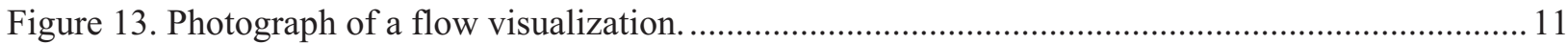

Figure 14. Time traces for various points in the flow for the three RSM turbulence model cases............ 12

Figure 15. $W$ profiles for the RSM cases for (a) y0u and (b) y0d compared to the MIR data (see

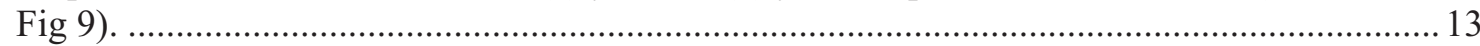

Figure 16. Contour plots for time mean velocity $W$ at $\mathrm{Z}=-70 \mathrm{~mm}$ for the Cases $1-3$, respectively......... 13

Figure 17. Profiles of time mean $U$ at $x 1 u, x 1 d, x 3 u, x 3 d, x 4 u, x 4 d, x 6 u, x 6 d$ for the three RSM cases.

Figure 18. Time traces for ensemble mean velocity $\langle u>$ at points $\mathrm{p} 5 \mathrm{~d}, \mathrm{p} 7 \mathrm{~d}$, and $\mathrm{p} 7 \mathrm{u}$ in the flow for the (a) standard $k \sim \varepsilon$ model, (b) AKN model, and (c) $k \sim \omega$ model. 16

Figure 19. Ratios of turbulent to molecular viscosity for the (a) standard $k \sim \varepsilon$ model, (b) the AKN $k \sim \varepsilon$ model, (c) the $k \sim \omega$ model and the (d) RSM model (Case 3)

Figure 20. Time mean velocity $W$ at (a) y0u and (b) y0d for eddy-viscosity Cases 4-6 compared to the MIR data.

Figure 21. Contour plots for time mean velocity $W$ at $Z=-70 \mathrm{~mm}$ for the (a) standard $k \sim \varepsilon$, (b) AKN, (c) $k \sim \omega$ eddy-viscosity models, and the (d) MIR data.

Figure 22. Profiles of time mean $U$ at $x 1 u, x 1 d, x 3 u, x 3 d, x 4 u, x 4 d, x 6 u, x 6 d$ for the 3 eddyviscosity cases..

Figure A-1. Top view of standard problem model geometry. 25

Figure A-2. Front view of standard problem model geometry. .26 


\section{TABLES}

Table 1. Percent difference of mass flows from measured rotameter values for the PIV-based flows. 6

Table 2. Summary of CFD model cases that employed the RSM turbulence model................................. 12

Table 3. Summary of CFD model cases that employed two-equation, eddy viscosity models. 14 


\section{ACRONYMS}

AKN Abe, Kondoh, and Nagano (turbulence model based on the $k \sim \varepsilon$ model and modified by researchers)

CFD computational fluid dynamics

DOE U. S. Department of Energy

INL Idaho National Laboratory

MIR matched index of refraction

NGNP next generation nuclear plant

PIV particle image velocimetry

RANS Reynolds-averaged Navier-Stokes

RSM Reynolds stress transport turbulence model

SST shear stress transport

TDR turbulent dissipation rate

TKE turbulent kinetic energy

URANS unsteady Reynolds-averaged Navier-Stokes

VHTR very high temperature reactor 


\section{Computational Fluid Dynamic Analysis of the VHTR Lower Plenum Standard Problem}

\section{INTRODUCTION}

The U.S. Department of Energy (DOE) is promoting the resurgence of nuclear power in the United States for both electrical power generation and production of process heat required for industrial processes such as the manufacture of hydrogen for use as a fuel in automobiles. The project DOE is funding to accomplish this is called the Next Generation Nuclear Plant (NGNP) Project, which is based on a Generation IV reactor concept called the very high temperature reactor (VHTR). The VHTR will use helium as the coolant at temperatures ranging from $450^{\circ} \mathrm{C}$ to perhaps $1000^{\circ} \mathrm{C}$. While computational fluid dynamics (CFD) has not previously been used for the safety analysis of nuclear reactors in the United States, it is being considered for existing and future reactors. It is fully recognized that CFD simulation codes will have to be validated for flow physics reasonably close to actual fluid dynamic conditions expected in normal and accident operational situations. To this end, experimental data have been obtained in a scaled model of a narrow slice of the lower plenum of a prismatic VHTR. The present report presents results of CFD examinations of these data to explore potential issues with the geometry, initial conditions, flow dynamics, and data needed to fully specify the inlet and boundary conditions; results for several turbulence models are examined. Issues are addressed and recommendations about the data are made.

The heated gas that has traversed the core of the VHTR enters a lower plenum where it mixes and then exits the reactor vessel. Figure 1 illustrates a plan view of the prismatic VHTR lower plenum showing numerous cylindrical support pillars, locations of jet inlets (various small circles), and the exit duct. There are concerns that the heated helium may create hot spots in the lower plenum of the reactor vessel because of differential heating of the helium in the core and that the helium will not be sufficiently mixed to attain a uniform temperature as it exits the reactor vessel. The application of a CFD code to characterize the helium flow in the lower plenum requires that the calculations be validated against experimental data that has a close relationship to the flow expected.

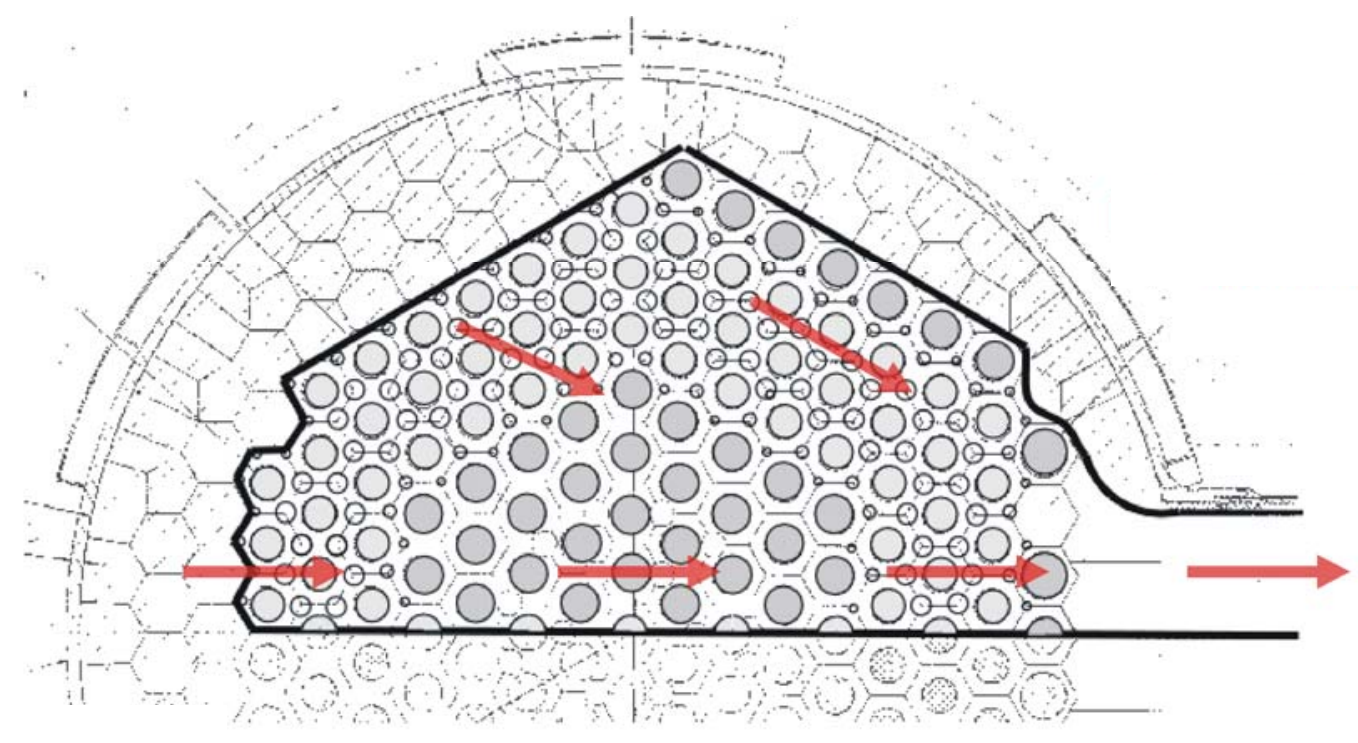

Figure 1. Plan view of the VHTR lower plenum. 
Experimental data have been taken using particle image velocimetry (PIV) in the INL's matched index of refraction (MIR) facility for a 1:6.55 scaled model of a narrow section of the lower plenum that is representative of the lower plenum flow. ${ }^{1}$ Figures 2 and 3 illustrate plan and isometric views of the scaled model, showing inlet ports, half and full support cylindrical posts, and the exit plane. The half cylinders are included in the scaled model because the model was designed to be an exact scaled replica of a slice of the lower plenum. The scaled model is $53.98 \mathrm{~mm}$ wide, $558.8 \mathrm{~mm}$ long (with the first jet located at $88.93 \mathrm{~mm}$ from the origin), and $217.5 \mathrm{~mm}$ high. The origin and coordinate axes are pictured in Figure 3. Diameters for the inlet jets and posts are $0.0221 \mathrm{~mm}$ and $0.03175 \mathrm{~mm}$, respectively. Full geometric details are given in Appendix A.

Inlet jets (open)

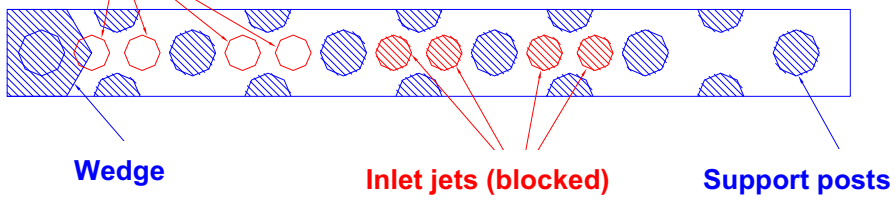

Figure 2. Plan view of the scaled model.

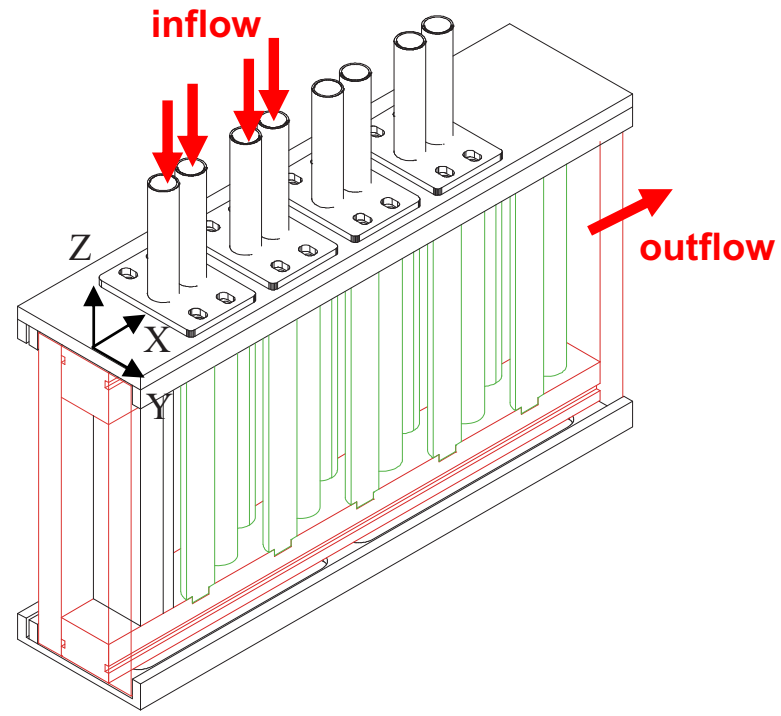

Figure 3. Isometric view of the scaled model and origin and coordinate axes for the CFD model.

The overall objective of the CFD study of the scaled model of the lower plenum has been to investigate issues related to the suitability of the experimental (MIR) data to be useful as a validation data set. Some of these issues are the suitability of the model geometry itself, how many inlet jets should be employed, how sensitive the internal flow is to the symmetry/asymmetry of the inlet jets, and how complex the flow actually is. Other issues include the completeness and accuracy of the inlet conditions of the four inlet jets and whether the flow inside the scaled model is unsteady because of vortex-shedding. Three-dimensional (3-D) CFD calculations have been carried out to investigate these issues for a jet inlet Reynolds number $\bar{u} d_{j e t} / v=12,400$, where $\bar{u}$ is the bulk velocity of the jet. The study investigates the use of Reynolds-averaged Navier-Stokes (RANS) turbulence models for the simulation of the MIR data. RANS turbulence models have been used extensively in various industries and been found to be the most practical approach to computing turbulent flows compared to large eddy simulation and direct numerical simulation approaches. 


\section{NONSTATIONARY TURBULENT FLUID DYNAMICS}

Turbulent flow is typically characterized by unsteady random fluctuations in all three spatial directions. In addition to these motions, there may be additional unsteady motions that are not random but are coherent. Such flows are termed nonstationary. Examples of these include turbulent flow in turbomachinery where there is unsteadiness from rotating blades, turbulent flow around bluff bodies that causes vortex-shedding, and turbulent flow that is being pumped by a pulsating pump. In the present case, it is assumed that the flow around the cylindrical posts in the scaled model generates vortices that shed from the downstream side of the posts and half-posts. For nonstationary turbulent flow, two averaging operations can be applied to the instantaneous flow quantities. ${ }^{2}$ First, the random turbulence is averaged out of the describing Navier Stokes equations by performing an ensemble average. Conceptually, this is done by considering that the flow is repeated many times and that the ensemble of results is simply averaged. The procedure is called Reynolds averaging and the resulting equations are called the RANS equations. A computational approach that employs the unsteady RANS equations is called a URANS approach. (For the case of stationary turbulent flow wherein the Navier Stokes equations are simply timeaveraged, the equations are also called the RANS equations and the approach using the RANS equations is called a RANS approach.) Mathematically, the instantaneous flow quantities for nonstationary turbulent flow are decomposed into an ensemble average and a (random) turbulent fluctuating component. For example, for the X-component velocity

$u=\left\langle u>+u^{\prime}\right.$

where $\langle u\rangle$ is the ensemble average and $u^{\prime}$ is the turbulent fluctuation, the ensemble average can vary in time because of nonturbulent unsteadiness. The ensemble-averaged quantity can be decomposed further into a time-averaged component and a coherent fluctuation. The coherent fluctuation is related to nonturbulent unsteadiness such as vortex-shedding. For example

$<u>=U+\tilde{u}$

where $U$ is the time average of the ensemble average (or long-time average or just time mean) and $\tilde{u}$ is the coherent fluctuation. These averaging processes are applied to the velocity components and pressure. The velocity components for the present study are $u, v$ and $w$ in the $\mathrm{X}, \mathrm{Y}$, and $\mathrm{Z}$ directions, respectively as shown in Figure 3. The CFD calculations for the turbulent flow in the scaled model presented herein compute the ensemble averages. These are concurrently time-averaged (by a running time-averaging calculation) because the experimental data (Ref. 1) are long-time averaged. Furthermore, the data were taken at a rate of 2-3 data planes per second, which is insufficiently rapid to capture the vortex shedding. However, the data were taken for time intervals exceeding 300 seconds such that a fairly representative long-time average should have been obtained.

\section{CFD MODELS}

\subsection{Outlet Boundary Conditions}

The scaled model shown in Figure 3, which is $104.8 \mathrm{~mm}$ wide, was positioned inside the test section of the INL's MIR facility. The cross section of the MIR test section is $609.6 \mathrm{~mm}$ square. The mineral oil working fluid not only flows into the scaled model, but around the outside of the model as well (at a different velocity than inside the model). Consequently, the oil from the outer flow joins the oil from the inner flow at the model exit. A concern regarding the flow in the scaled model was that the outer flow would alter the flow at the exit of the scaled model, possibly affecting the flow inside the model. A twodimensional study, using a URANS approach, was performed to assess the effects of the outer flow on the inner flow to determine whether the outer flow would need to be included in the CFD model. ${ }^{3}$ The commercial code FLUENT ${ }^{4}$ was used in the 2-D study. The Reynolds stress transport turbulence model 
(RSM) was employed. Full details, including the achievement of mesh and iterative convergence, are provided in Ref. 3.

Figure 4 illustrates the geometry and streamlines for the 2-D case of including the flow inside the scaled model as well as the flow around the outside of the scaled model but within the MIR test section (called the 'inner-outer' case). As can be seen in Figure 4, the flow developed shedding vortices around the full and half cylinders inside the flow geometry representing the inside of the scaled model; it can also be seen that there are vortices forming behind the $25.4 \mathrm{~mm}$ thick walls of the scaled model. It was found that because of the vortex shedding from the last cylinder there is inflow at the exit plane of the scaled model.

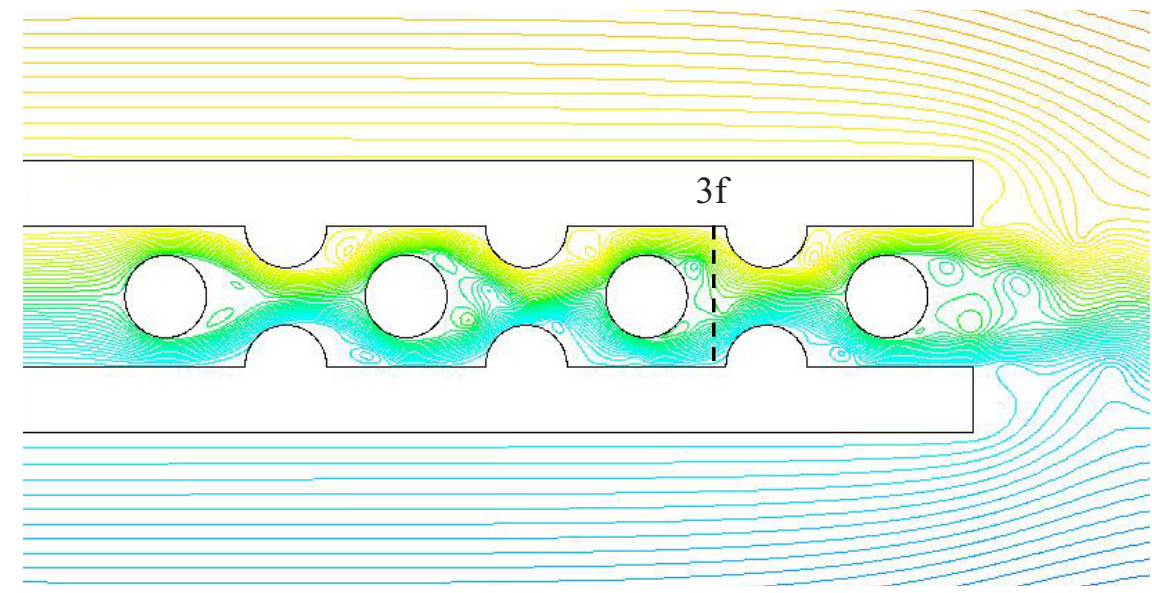

Figure 4. Stream function contours of the flow in the CFD model, which includes the outer flow showing vortex-shedding behind the physical model in the MIR test loop.

Additional 2-D cases were run where only the inner flow was computed and two different boundary conditions were tried at the outlet plane of the inner flow (coincident with the ends of the model walls; see Figure 4). The two boundary conditions used were the 'outflow' and 'pressure-outlet' conditions. These same two boundary conditions are available in both commercial CFD codes FLUENT (Ref. 4) and STARCCM.$+{ }^{5}$ The outflow boundary condition enforces a constant gradient condition for the velocity components, while the pressure-outlet condition sets a constant static pressure just downstream of the outlet plane. Actually, neither of these conditions is exactly correct because the outlet to the inner flow domain is just barely downstream of the last full post such that vortex shedding occurs at the outlet.

Figure 5 illustrates the time mean velocity $U$ at the outlet plane and at profile location $3 \mathrm{f}$ (see Figure 4), for the cases employing the outflow and pressure-outlet boundary conditions at the scaled model outlet plane compared to the reference case of including the outer flow in the CFD domain. As can be seen, the three cases are distinctly different at the outlet plane, but the pressure-outlet case compares very closely with the reference case at profile location $3 \mathrm{f}$. Figure 6 compares the three cases for the time mean velocity $V$ and the kinematic Reynolds stress $\bar{u} \bar{v}$ at location $3 \mathrm{f}$. As can be seen, the pressure-outlet results closely match those for the reference case. Furthermore, the results plotted in Figures 5 and 6 for the outflow boundary condition are significantly different from the reference case, recommending that this boundary condition not be used for the outlet boundary.

It is clear that while the results for the pressure-outlet condition are not accurate near the outlet, they are very close to the results of the reference case further upstream. These results provide confidence that 
using only the flow domain inside the scaled model for the 3-D case along with a pressure-outlet boundary condition will not cause inaccuracy in the results, except in the region close to the outlet plane.

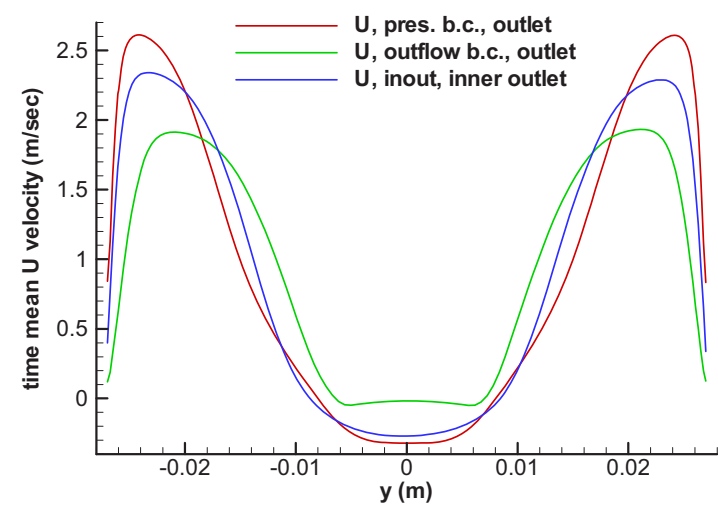

(a)

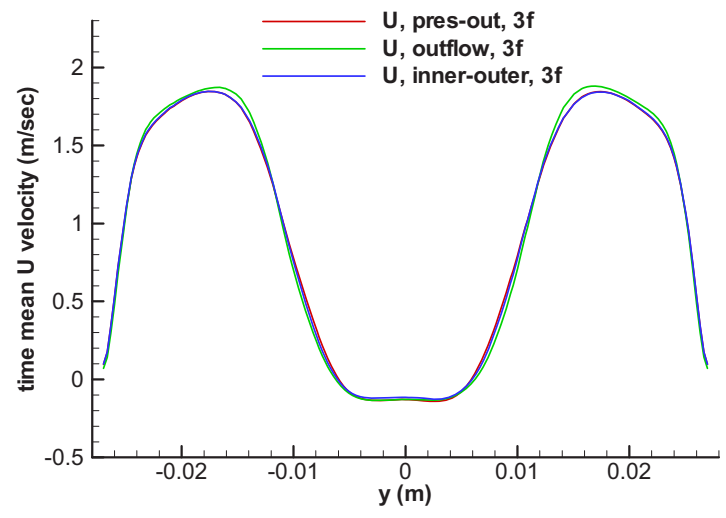

(b)

Figure 5. Profiles of the time mean velocity $U$ at the (a) outlet plane of the scaled model and (b) profile location $3 \mathrm{f}$ (see Figure 4), for cases using pressure-outlet and outflow boundary conditions compared to the reference inner-outer case.

(a)

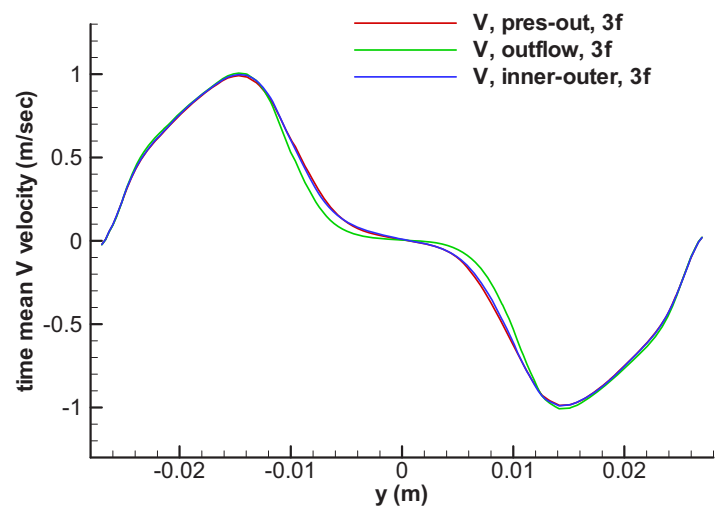

(b)

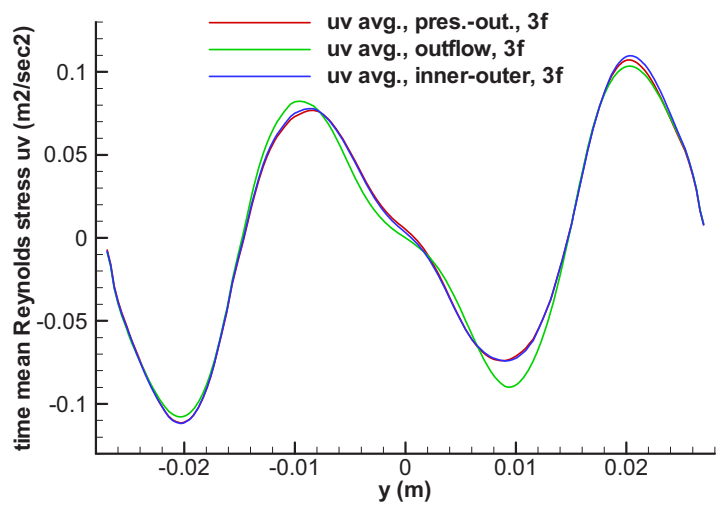

Figure 6. Profiles of the (a) time mean velocity $V$ at profile location $3 f$ (see Figure 4) and (b) Reynolds stress $\bar{u} \bar{v}$ at $3 \mathrm{f}$ for cases using pressure-outlet and outflow boundary conditions compared to the reference inner-outer case.

\subsection{Inlet Boundary Conditions}

Accurate and complete inlet boundary conditions are critical to the usefulness of any CFD validation data set. These include the velocity components and turbulence parameters, usually the turbulent kinetic energy (TKE). The inlet mass flows for the MIR facility were measured by mass flow rotameters with each inlet duct having its own rotameter. The inlet mass flows were physically adjusted based on these rotameters. The mass flow to the first jet was adjusted to be two-thirds of the others because the flow area is two-thirds that of the others (see Figure 2). The rotameters provide accurate mass flow rates. During the course of the data taking, PIV data were taken for a vertical stretch of the inlet ducts above the jet inlet plane of the scaled model. These data were taken in planes illuminated by laser sheets that were normal to the Y direction, the same as for all the PIV data. Eleven planes of PIV data were taken. Threedimensional velocity data were taken in these illuminated laser sheet planes, which were approximately 
$2 \mathrm{~mm}$ in width. The data were then processed by the PIV software and velocity information produced. Data at $Z=9.7 \mathrm{~mm}$ and $Z=11 \mathrm{~mm}$ were extracted for use as inlet conditions.

The inlet data were configured into tables that could be read by the STARCCM+CFD code. The data were interpolated onto the grid that was created and could then be integrated by the code to obtain the mass flow rates. As it turns out, the reduced PIV data that were integrated by STARCCM+ do not generally compare well with the mass flow rotameter data. Figure 7 visually compares the mass flow rates for the four jets based on the rotameter flow rates and the integrated PIV data at 9.7 and $11 \mathrm{~mm}$. As can be seen, the mass flow rates for the PIV data are all lower than those from the rotameters. While each jet profile could be multiplied by a specific factor to obtain the same mass flows as each rotameter, it seems that this approach becomes too far removed from using the actual MIR data. Therefore, the vertical velocity data points for the $11 \mathrm{~mm}$ PIV data were all multiplied by one factor to yield the same total inlet mass flow rate as for the uniform inlet case. The flow rates for these modified $11 \mathrm{~mm}$ data are also shown in Figure 7. Table 1 gives the percentage differences between the mass flows, based on the PIV data, and the rotameter data. The rotameter data are considered to be the most accurate inasmuch as they are derived directly from measurements taken by the calibrated instruments.

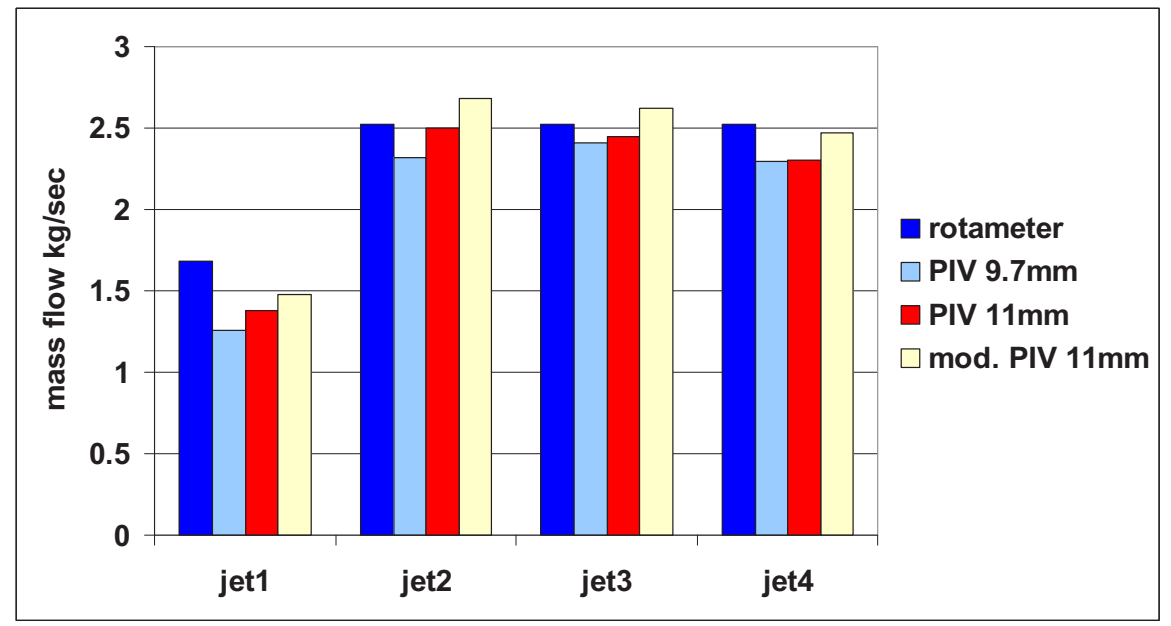

Figure 7. Comparison of mass flow rates for the four inlet jets derived from rotameter data and detailed PIV measurements at $\mathrm{Z}=9.7$ and $11 \mathrm{~mm}$ and the modified PIV data at $11 \mathrm{~mm}$.

Table 1. Percent difference of mass flows from measured rotameter values for the PIV-based flows.

\begin{tabular}{|l|l|l|l|l|l|}
\hline & \multicolumn{1}{|c|}{ Jet 1 } & \multicolumn{1}{c|}{ Jet 2 } & \multicolumn{1}{c|}{ Jet 3 } & \multicolumn{1}{c|}{ Jet 4 } & \multicolumn{1}{c|}{ Total } \\
\hline PIV $9.7 \mathrm{~mm}$ & $25.1 \%$ low & $7.98 \%$ low & $4.46 \%$ low & $8.99 \%$ low & $10.4 \%$ low \\
\hline PIV $11 \mathrm{~mm}$ & $18.1 \%$ low & $0.78 \%$ low & $2.99 \%$ low & $8.80 \%$ low & $6.70 \%$ low \\
\hline mod. PIV $11 \mathrm{~mm}$ & $12.2 \%$ low & $6.37 \%$ high & $4.00 \%$ high & $2.23 \%$ low & $0.0 \%$ \\
\hline
\end{tabular}

Clearly, there are some significant differences between the rotameter data and the mass flows based on integration of the detailed PIV data, in particular for Jet 1. Even the modified $11 \mathrm{~mm}$ PIV data are still $12 \%$ low. Greater confidence in the PIV-measured inlet data would be obtained if the differences from the rotameter data were less than 5\%. This is actually true for Jet 3 at 9.7 and $11 \mathrm{~mm}$ and Jet 2 at $11 \mathrm{~mm}$. However, there are also significant differences between the PIV inlet data from 9.7 to $11 \mathrm{~mm}$ for Jets 1 and 2, which casts further doubt on the accuracy of the PIV-based inlet data. It would be much better if the PIV data could be taken from laser sheets illuminating the jet inlet ducts in the plane of the crosssections of the ducts because the data would be much denser, therefore providing a much more detailed profile of the flow. 
Calculations have been performed using all of the inlet conditions represented by the four cases in Figure 7. In the case of the PIV data based conditions, the turbulent kinetic energy is also provided. In the case of the inlet conditions based on the rotameter data, the velocity component normal to the inlet flow plane is given as uniform; the other velocity components are given as zero. The turbulent kinetic energy is also given as a constant. Finally, the additional turbulence quantity required by the CFD codes, which is typically not a measurable quantity such as turbulent dissipation rate, is simply estimated or the default value is used. Details are given in the results section for each case.

\subsection{Meshing}

In the previous 2-D study by the author (Ref. 3), three different grids of increasing fineness were employed to check for grid independence. It was found that the intermediate and finest grid yielded very similar results. Initially, for the 3-D study, two 2-D grids were constructed based on the coarsest and intermediate grids from the 2-D study (Ref. 3). The meshes were then extruded in the vertical direction to obtain full 3-D grids. Additional inlet ports about $9.7 \mathrm{~mm}$ high were added for the inlet jets. Inlet data were provided at this level for the velocity components and the turbulent kinetic energy. The number of cells allocated in the third dimension is 15 for the inlet jets and 80 and 125 in the body of the model for the coarser and finer meshes. The mesh cells in the 3-D grid are hexahedral. The two 3-D meshes contain about 4 million cells (grid a2) and 14 million cells (grid b2), respectively. The commercial mesh software GAMBIT 2.4.6, which is bundled with FLUENT (Ref. 4), was used to create the meshes. Two other meshes were also constructed as computations made some of the problems with the inlet conditions clear. The third mesh (grid a3) is the same as grid a2 except that the inlet ports are $11 \mathrm{~mm}$ high inasmuch as alternative inlet data were provided at this location. The fourth mesh was constructed to have inlets that are $88.5 \mathrm{~mm}$ high. This height corresponds to the location of screens that were placed in the inlets of the actual scaled model to generate turbulence in the MIR tests. Flow straighteners are placed upstream of the screens to straighten the flows after 90 degree bends in the inlet supply ducts. This fourth grid (grid a4) has 100 cells in the Z-direction in the inlet channels and 110 cells in the Z-direction for the main body of the model. Grid a4 has a total of 5.78 million cells. The relatively coarser grids, a2-a4, were employed to see if differentiations can be made among the several variations in initial and inlet conditions and turbulence model. Comparisons can later be made with the finer grid to show the effects of the coarser gird. Also, a full 5 seconds of simulation time takes about 2 weeks for the coarser grids using on the order of 150 cpus. The finer grid takes much longer. Figure 8 illustrates the bottom wall plane of the coarser grid showing two of the inlet ports and some of the half- and full-posts and an isometric view of the 3-D a2 grid. The inlet jet ports are shown in green, the outlet plane in red, and the walls in gray.

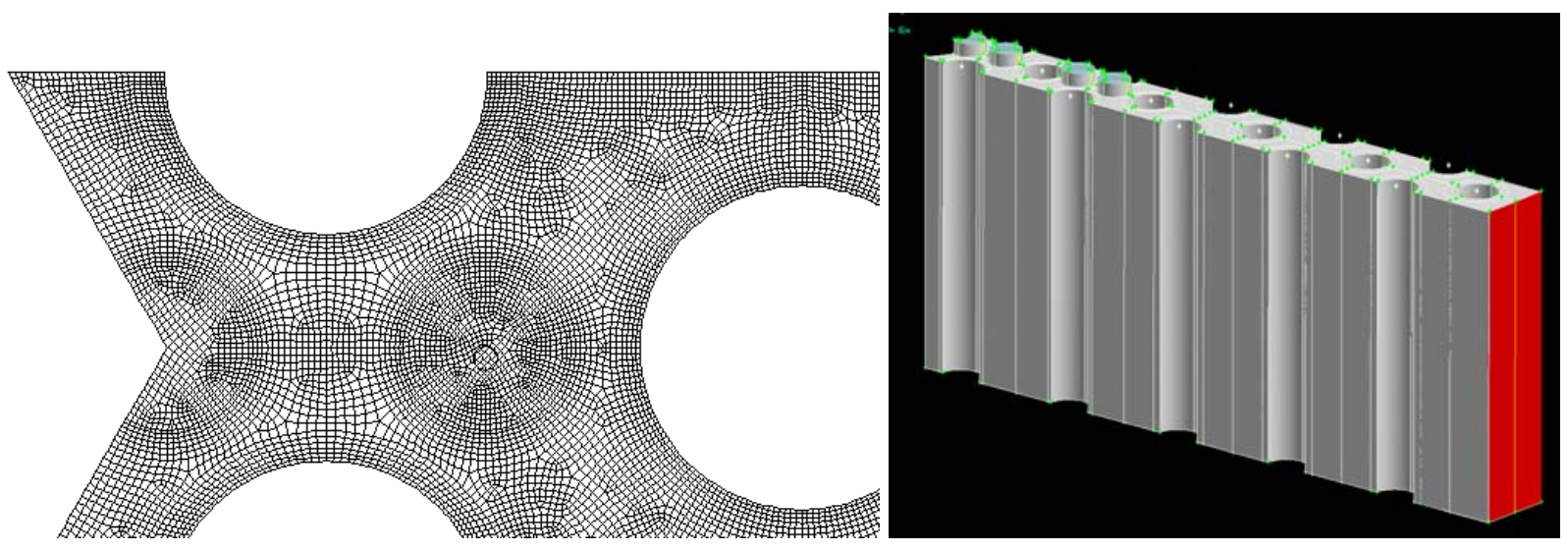

Figure 8. A view of the bottom plane of the a3 grid and an isometric view of the same grid showing inlet ports (green), outlet plane (red), and walls (gray). 


\subsection{Computational Details}

The commercial CFD code STAR-CCM+ (Ref. 5) is used for almost all of the 3-D computations because a large number of parallel licenses are available. The flow problem is set up using a URANS approach because of vortex shedding, as in the 2-D study (Ref. 3). Second-order differencing is used for both spatial and the implicit temporal discretization; the segregated solver is used. Walls are considered smooth and are assigned the no-slip condition.

The initial simulations using the 14-million-cell mesh use a time step of $7.5 \times 10^{-5} \mathrm{sec}$, which was found to be sufficiently small in the 2-D study (Ref. 3). Simulations using the coarser grids (a2-a4) use a time step of $2.0 \times 10^{-4}$. The pressure-outlet boundary condition is used at the outlet as discussed above. The iterative convergence for each time step was set based on the calculation of a Poiseuille flow, which has an analytical solution. It was found from the Poiseuille flow calculation that a residual computed in STARCCM+ in the default mode is converged at a value of about $2 \times 10^{-4}$.

Figure 9 illustrates profile locations in the scaled model that will be used for data comparisons. The profiles shown in the plan view each represent two profiles: one at $Z=-70 \mathrm{~mm}$ (given suffix ' $u$ ') and one at $Z=-150 \mathrm{~mm}$ (suffix ' $\mathrm{d}$ '). So for example, profile $x 1 \mathrm{u}$ is at location $\mathrm{x} 1 \mathrm{at} Z=-70 \mathrm{~mm}$, while $\mathrm{p} 5 \mathrm{~d}$ is at point $\mathrm{p} 5$ at $Z=-150 \mathrm{~mm}$; see Figure 3 .

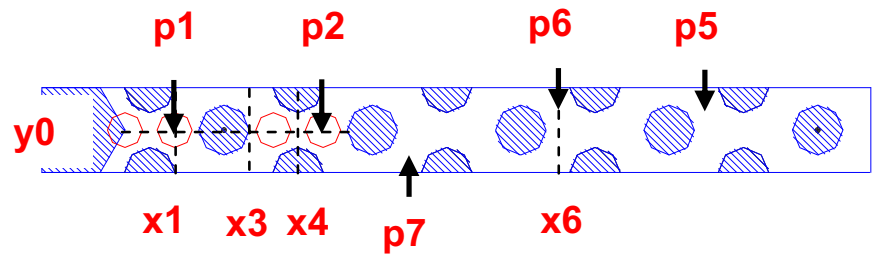

Figure 9. Locations of profiles and points for data comparison.

The CFD calculations must be long-time averaged to compare with the MIR data, which are averaged over about 300 seconds. The long-time averaging is computed concurrently with unsteady calculations. The question arises as to how long the simulation must be carried out to provide an unchanging long-time average. Figure 10 plots the time mean velocity $W$ along profile y0u (see Figure 9), at five different points in time for the case of employing grid a4, the shear-stress transport (SST) $k \sim \omega$ turbulence model and uniform inlet profiles, which captures the four jet inlets along the centerline. As shown, computations for 2.4 seconds or less are significantly different from longer time averages in the region of the first jet $(\mathrm{X}<0.115 \mathrm{~m})$. At 3 seconds and beyond, changes are small, especially for the regions beyond the first jet.

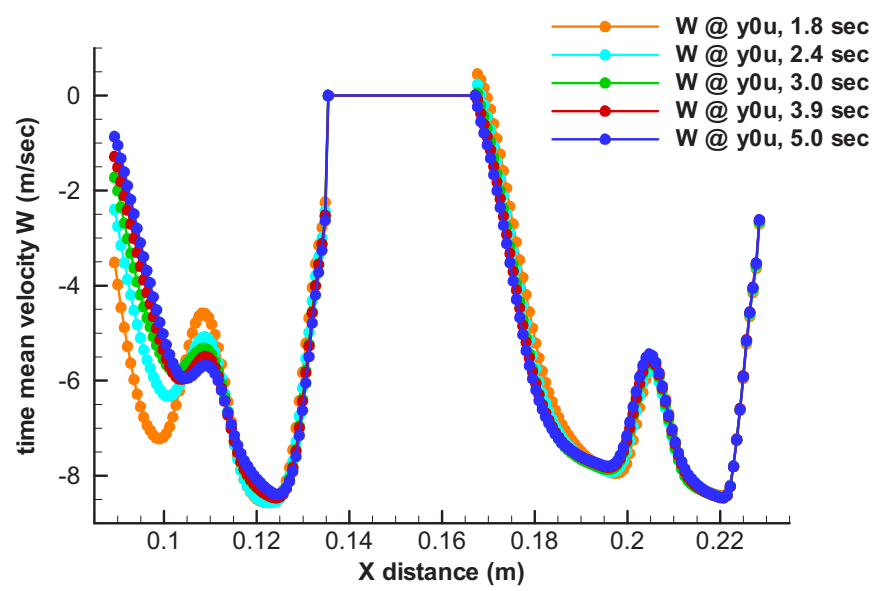

Figure 10. Time mean velocity $W$ along data profile ' $y 0 u$ ' for five different time intervals. 


\subsection{Initial Conditions}

Two methods for beginning the unsteady calculations were used and investigated. The first method is to begin a steady calculation until the residuals stop decreasing, then initiate the unsteady calculations. The second is to start from a stagnant condition in the body of the model. Computations were made using the Abe-Kondoh-Nagano (AKN) $k \sim \varepsilon$ turbulence model starting from these two initial conditions. Figure 11 compares results at about 3 seconds for these two cases to the MIR data and another case wherein the RSM is used. There are some relatively minor differences in the results computed using the two different initial conditions, especially compared to differences related to the turbulence model. These differences may simply be because the long-time averages for the stagnant case includes some initial transient values that are not included in the other case.
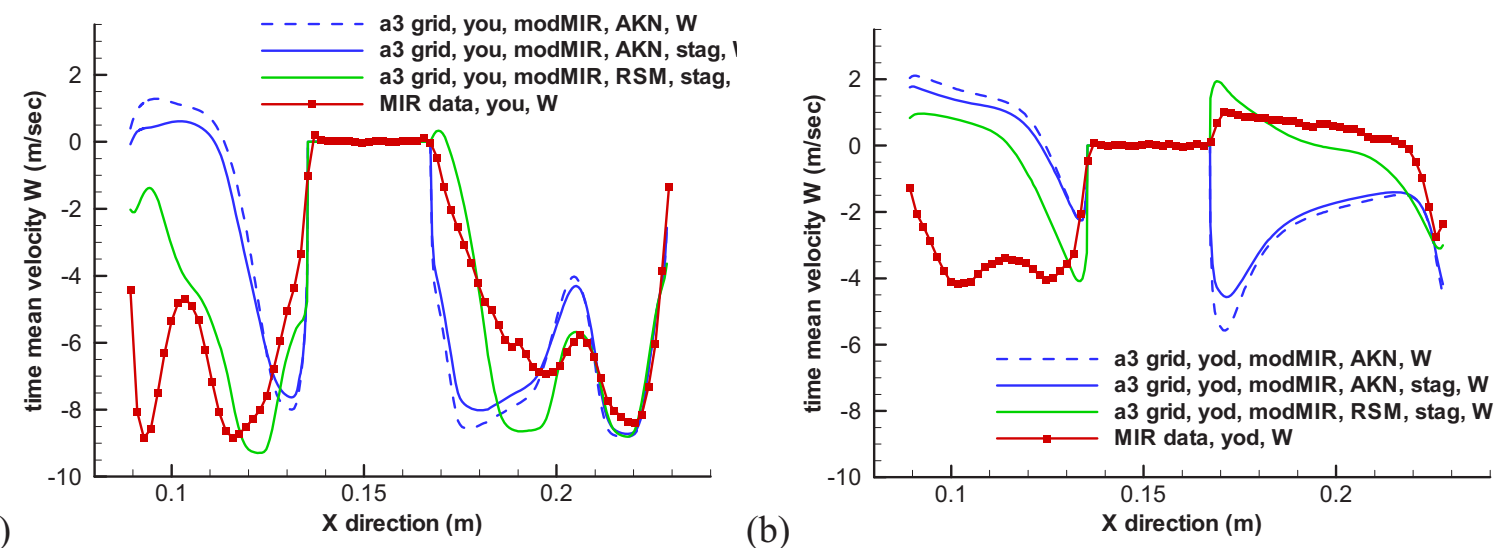

(a)

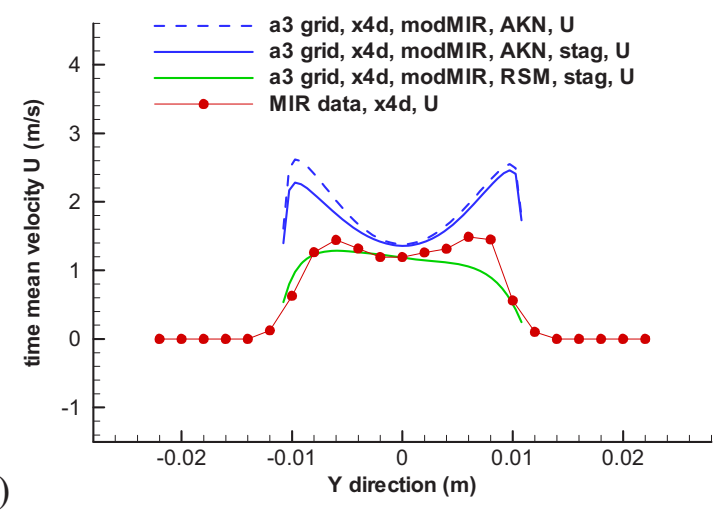

(b)

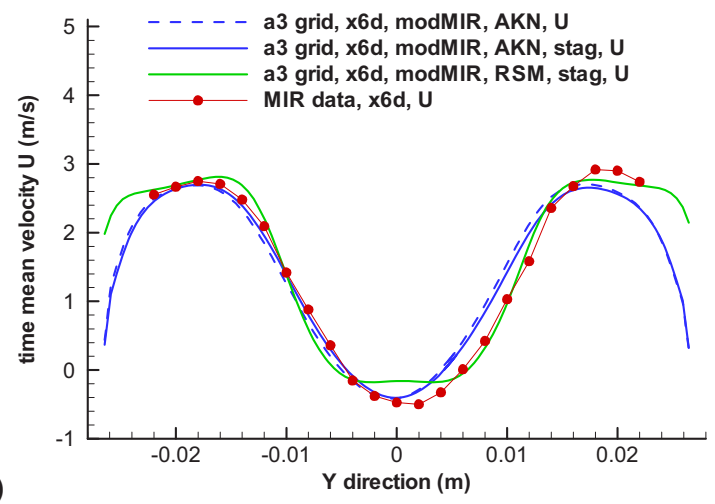

Figure 11. Comparisons of results for the AKN turbulence model starting from a semiconverged steady initial condition and from a stagnant condition to results for the RSM from stagnant initial conditions and the MIR data for (a) W at y0u, (b) W at y0d, (c) $\mathrm{U}$ at $\mathrm{x} 4 \mathrm{~d}$, and (d) $\mathrm{U}$ at $\mathrm{x} 6 \mathrm{~d}$.

\subsection{Turbulence Models}

The flow in the scaled model of the lower plenum is designed to be turbulent because the flow in the actual lower plenum will be turbulent. There must be some way to account for the effects of turbulent transport of momentum. The most practical way is to use a RANS turbulent model because the compute time is much less than for more sophisticated approaches, including large eddy simulation and direct numerical simulation. There are several RANS turbulence models available in the commercial CFD codes that have been employed at INL. There are dozens of RANS turbulence models in the fluid dynamics literature that have been devised and revised over the years. The investigation of the performance of a RANS turbulence model is one of the most critical tasks necessary to be able to validate a CFD analysis. 
The turbulence model will have a very significant effect on the results obtained. Any turbulence model used for reactor safety analysis must be validated for the range of phenomena to which it is to be applied.

The behavior of the turbulence model in the near-wall region is very important because this region determines wall friction and heat transfer. The region adjacent to the wall is actually nonturbulent because the zero velocity boundary condition forces the flow to be viscous. This viscous region has been experimentally found to be a region for which a dimensionless wall distance parameter $y^{+}=y u_{\tau} / \rho<11$. Here $y$ is the dimensional distance, $u_{\tau}$ is the friction velocity defined as the square root of the wall shear stress divided by the density, and $\mu$ is the molecular viscosity (Refs 4 and 5). Some turbulence models require the additional specification of a wall treatment while others are designed to intrinsically apply all the way to the wall. The near-wall $y^{+}$values for the grids used herein are mostly between 1 and 12 , putting them in the viscous region or sublayer. This is appropriate for turbulence models that are designed to handle the viscous sublayer. However, standard wall functions, which are recommended for use for the near-wall node having a $y^{+} \geq 25$ are also designed to work within the viscous sublayer. It was found that the application of the RSM for the tube bank flow (Ref. 2) using wall functions, but with a near-wall $y^{+}$in the viscous sublayer gave good results for the time mean streamwise velocity. The RSM with the linear pressure-strain option is used herein with both the finer 14 million cell mesh and the coarser 4 million cell mesh. The all $y^{+}$wall model (STARCCM+) is used, which is equivalent to wall functions (as designated in FLUENT).

In addition, three two-equation eddy viscosity RANS turbulence models are employed to investigate their performance for the lower plenum problem. These are the standard $k \sim \varepsilon$ two layer model with the all

$y^{+}$wall model, the AKN $k \sim \varepsilon$ low Re model with the all $y^{+}$wall model and the Menter SST $k \sim \omega$ model with the all $y^{+}$wall model. The latter two models are specifically designed to apply to within close proximity of the wall $\left(y^{+}<11\right)$, while the standard $k \sim \varepsilon$ model breaks the wall region into two layers, a viscous sublayer and a turbulent sublayer. Documentation for the actual equations employed for the above turbulence models is provided in the user manuals for STARCCM+ (Ref. 5).

\section{RESULTS AND DISCUSSION}

Results for the CFD model computations are presented in this section. First, results are presented that examine the main flow features present in the computations and in a visualization study. Detailed results are then presented to compare the calculations with the MIR data to investigate the results for varying the initial conditions, the inlet conditions and the turbulence model used to represent the turbulence.

\subsection{Major Flow Features}

Figure 12 illustrates a 3-D streamline computation from initial points located at the four jet inlets for the AKN $k \sim \varepsilon$ model at 3.14 seconds. The view of the figure is reversed from previous geometrical presentations because of the view of the visualization study. Figure 13 is a photograph of a flow visualization test made by injecting air into Jet 1 (at the far right) with mineral oil flowing into all four jets. It can be seen that the major flow features are present in the streamline figure that are present in the actual flow including a large recirculation region in the upper center of the flow, a smaller recirculation region at the lower right, a recirculation zone between Jets 2 and 3, and flow that ascends gradually toward the left after moving beyond the jets. 


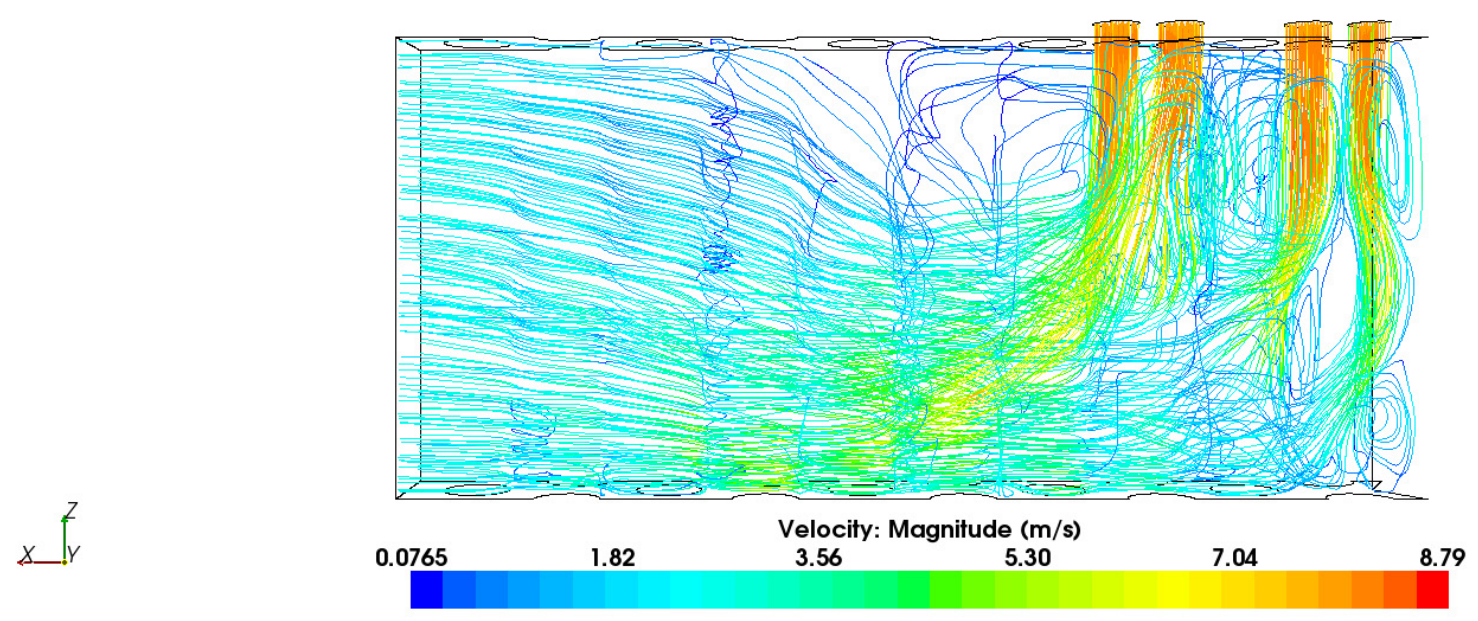

Figure 12. 3-D Streamlines for AKN model at $3.14 \mathrm{sec}$.

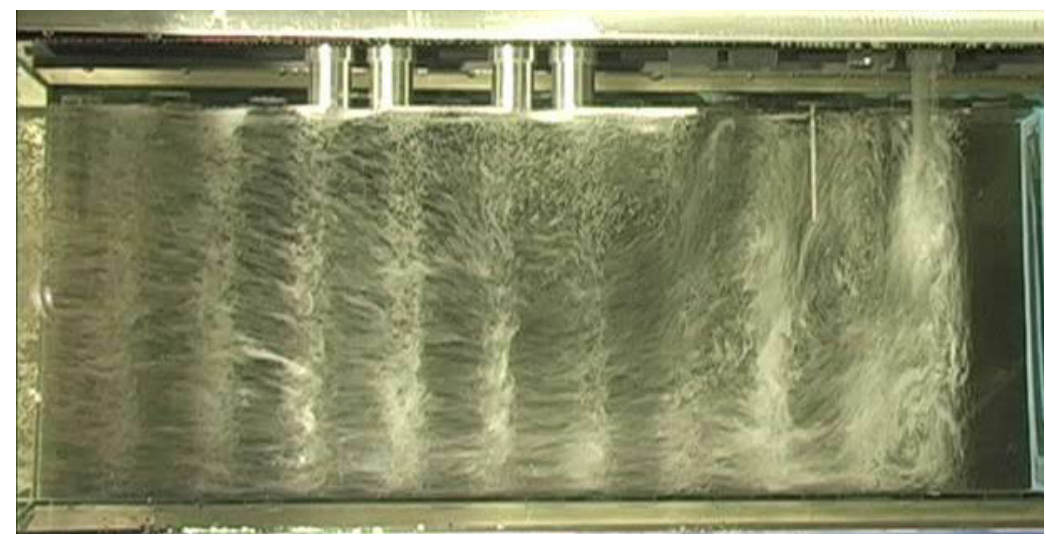

Figure 13. Photograph of a flow visualization.

\subsection{Turbulence Models and Inlet Conditions}

\subsubsection{Reynolds Stress Transport Model}

Results are obtained for the most sophisticated RANS turbulence model available in the commercial CFD code STARCCM+, the RSM. A differential transport equation is solved for each of the unique Reynolds stresses $\left(-\rho \overline{u_{i}^{\prime} u_{j}^{\prime}}\right)$ that appear in the RANS equations of fluid dynamics. All three cases computed employ the high $y^{+}$wall treatment, which is equivalent to wall functions. The first case employs the finer grid $\mathrm{b} 2$ with uniform inlet conditions at $Z=9.7 \mathrm{~mm}$. The inlet $Z$-direction velocity $W$ is set to the bulk velocity values that are based on the mass flow rotameter measurements as discussed above, that is $(7.922,7.918,7.918,7.918) \mathrm{m} / \mathrm{sec}$ for Jets $1-4$, respectively; the other velocity components are set to zero. The turbulent kinetic energy and turbulent dissipation rates are set to $0.01 \mathrm{~J} / \mathrm{kg}$ and $0.1 \mathrm{~J} / \mathrm{kg}$-sec, respectively for all of the jets. This case was computed for 9 seconds of simulation time. Initially, the time step was set to $7.5 \times 10^{-5} \mathrm{sec}$., but was later increased to $5.0 \times 10^{-4} \mathrm{sec}$. because the flow was becoming steady. The second RSM case also used grid b2, but used unmodified MIR inlet data at $9.7 \mathrm{~mm}$ for the velocity components and the turbulent kinetic energy. The turbulent dissipation rate (TDR) for Case 2 is set to $0.1 \mathrm{~J} / \mathrm{kg}$-sec for all of the jets. Case 3 used the coarser a3 grid and employed the modified MIR inlet data at $\mathrm{Z}=11 \mathrm{~mm}$ for the velocity components and the turbulent kinetic energy. The TDR is set to $1.0 \mathrm{~J} / \mathrm{kg}$-sec for all of the jets. These cases are summarized in Table 2. 
Table 2. Summary of CFD model cases that employed the RSM turbulence model.

\begin{tabular}{|c|c|c|c|l|l|c|c|}
\hline Case & $\begin{array}{c}\text { Turbulence } \\
\text { Model }\end{array}$ & $\begin{array}{c}\text { Wall } \\
\text { Model }\end{array}$ & Grid & \multicolumn{1}{|c|}{ Inlet Velocity } & $\begin{array}{c}\text { Inlet TKE } \\
(\mathbf{J} / \mathbf{k g})\end{array}$ & $\begin{array}{c}\text { Inlet TDR } \\
(\mathbf{J} / \mathbf{k g}-\mathbf{s e c})\end{array}$ & $\begin{array}{c}\text { Simulation } \\
\text { Time (sec) }\end{array}$ \\
\hline 1 & RSM & high $y^{+}$ & b2 & Uniform & 0.01 & 0.1 & 9.1 \\
\hline 2 & RSM & high $y^{+}$ & b2 & MIR 9.7 & MIR 9.7 & 0.1 & 4.6 \\
\hline 3 & RSM & high $y^{+}$ & a3 & Mod. MIR 11 & Mod. MIR 11 & 1.0 & 2.4 \\
\hline
\end{tabular}

The unsteady nature of the numerical solution can be investigated by plotting time evolution curves for the ensemble velocity at various points. Figure 14 plots ensemble mean velocities $\langle u\rangle$ and $\langle w\rangle$ at various points for the three cases. Figure 9 shows the point locations. The time traces for Cases 1 and 2 indicate that steady state is reached or nearly reached; Case 3 exhibits low-amplitude oscillations. Additionally, a steady-state solution was obtained for a case the same as Case 1, except assuming that the flow was steady; the two solutions are the same. This is in contrast with the 2-D study performed to investigate outlet boundary conditions as discussed above (Ref. 3). There, the RSM was used and the 2-D flow was clearly nonstationary with many shedding vortices (see Figure 4). As will be shown below, results for the three, two-equation eddy viscosity turbulence models exhibit unsteadiness with significant variations in amplitude. It is not clear why the RSM tends toward a steady solution for the 3-D cases. Unfortunately, the frequency of the MIR PIV data was only two to three data sheets per second, which is too slow to capture the vortex shedding, which was on the order of 0.03 seconds (Refs. 3 and 7).

(a)

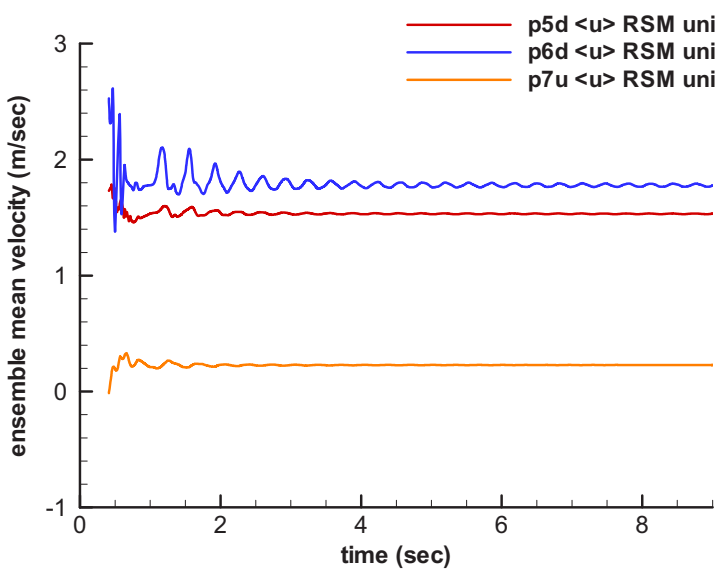

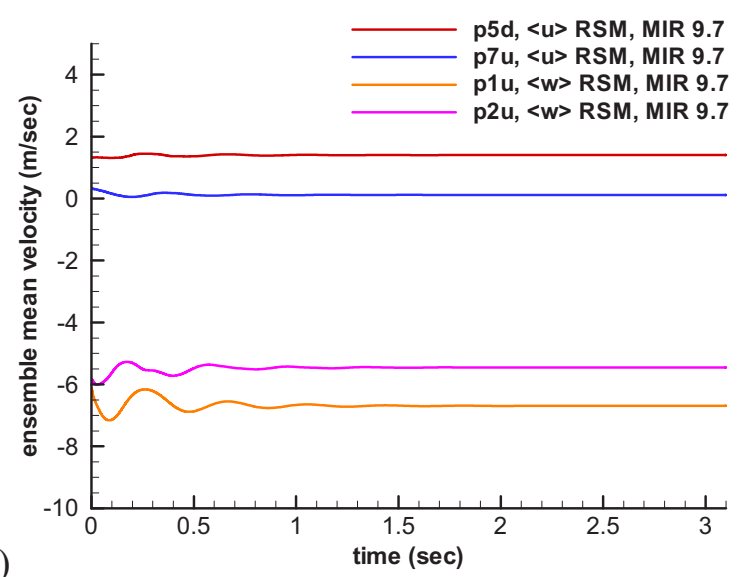

(b)

p5d $<u>$ RSM modMIR11 p7 $\mathrm{u}<\mathrm{u}>\mathrm{RSM}$ modMIR11 p1 $u<w>$ RSM modMIR11 p2u $<w>$ RSM modMIR1

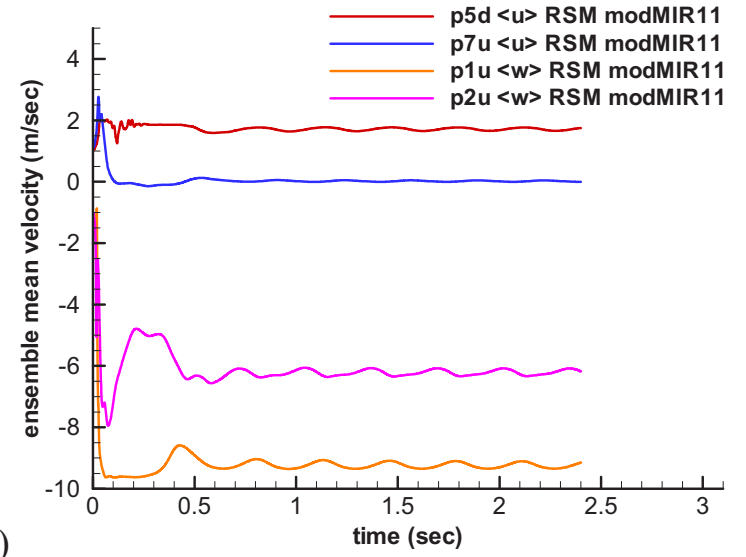

(c)

Figure 14. Time traces for various points in the flow for (a) Case 1, (b) Case 2 and (c) Case 3. 
Figure 15 illustrates time mean velocity $W$ profiles for the three RSM cases compared to the MIR data along profiles y0u and y0d (see Figure 9). The time mean velocity is equivalent to long-time averages and is appropriate for comparison with the MIR data as the data are long-time averaged. Signatures of the four inlet jets are clearly visible in the MIR data for y0u. The uniform inlet case does show a signature for Jet1 with a magnitude of about $5.8 \mathrm{~m} / \mathrm{sec}$, albeit farther to the right than the data, while the two cases that employ MIR data for the inlet show the first jet a little too far to the left with magnitudes of only $2 \mathrm{~m} / \mathrm{sec}$. For profiles at y0d, the MIR data exhibit signatures for the first two jets. The calculations, however, miss the first and show the second further downstream. Overall, the results for the uniform inlet conditions, which have the correct inlet mass flows, show the best qualitative and quantitative results.

(a)

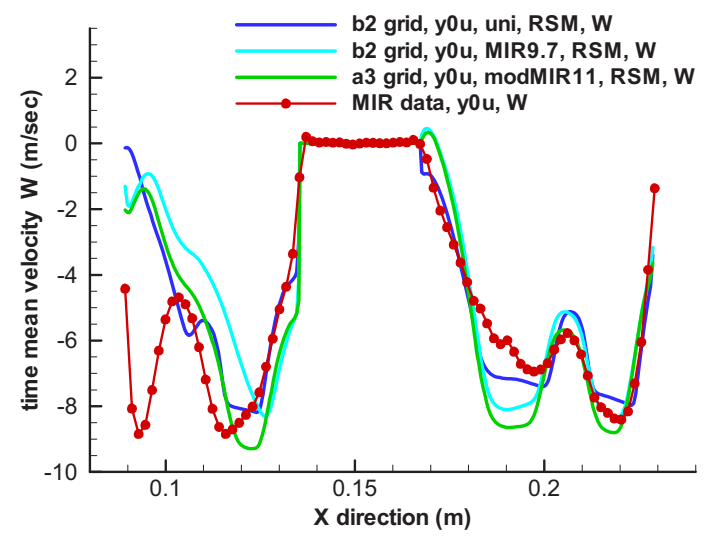

(b)

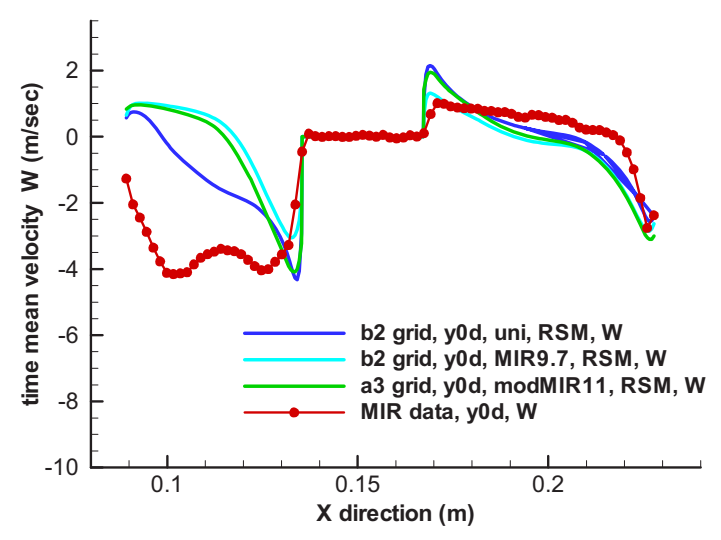

Figure 15. $W$ profiles for the RSM cases for (a) y0u and (b) y0d compared to the MIR data (see Fig 9).

More can be learned from contour plots at the same $\mathrm{Z}$ distance $(\mathrm{Z}=-70 \mathrm{~mm})$ for the same time mean velocity $W$, as for Figure 15a. Figure 16 shows contour plots for the three RSM cases at this $Z$ location.

(a)
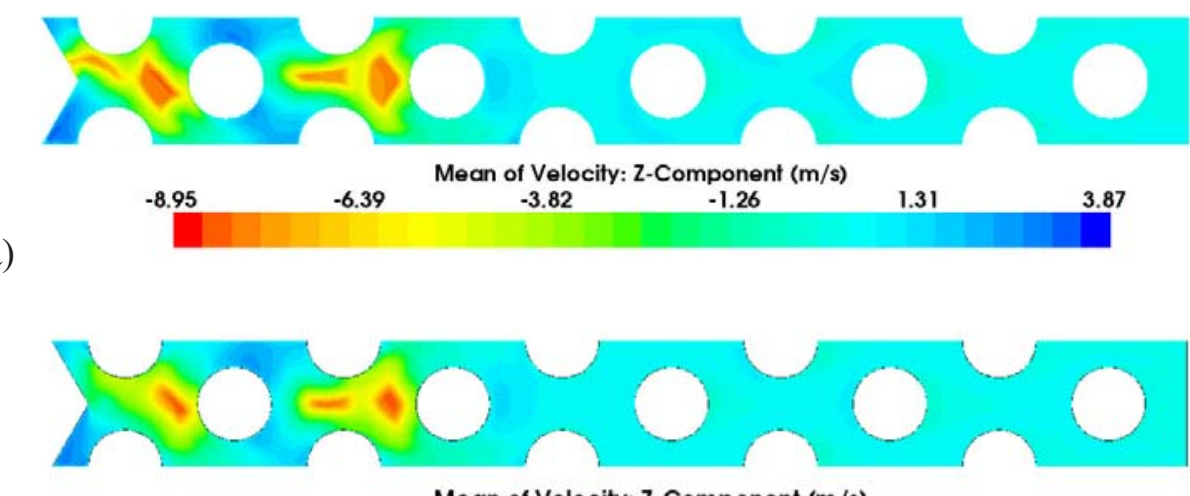

(b)
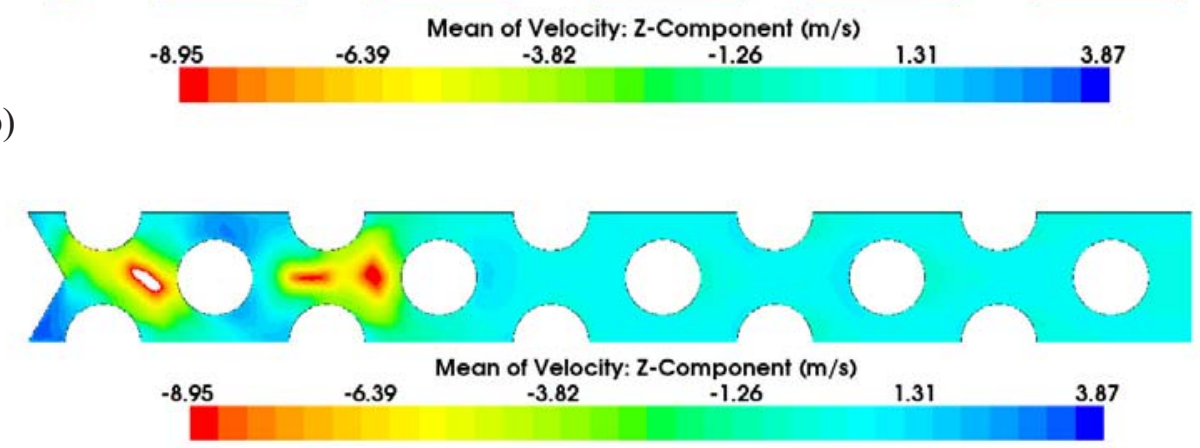

(c)

Figure 16. Contour plots for time mean velocity $W$ at $\mathrm{Z}=-70 \mathrm{~mm}$ for (a) Case 1, (b) Case 2, (c) Case 3. 
It is clear that significant asymmetry is present in all three cases in the vicinity of the first two jets, though the flow in the region of the third and fourth jets is quite symmetric. The fact that the first jet appears for Case 1, Figure 16a, but only for Case 1, is apparently due to the fact that the inlet mass flow for Jet 1 for this case is correct, while the inlet mass flow for the other two cases is too low (see Tables 1 and 2). The fact that the first jet is skewed to one side at this location may be related to the presence of the wedge in the scaled model. That is, there could be a semistable state of asymmetric flow that is caused by the presence of the wedge, which separates the flow into two regions. Or it could be that there is something in the nature or even the coding of the RSM in STARCCM+ that is leading to an asymmetry here.

The presence of the wedge causes there to be a wall jet for the first jet because of its location. It seems that this is a separate effect that could be investigated separately from the rest of the flow field of the lower plenum. It may be the case that the wall-jet flow is not of concern to the final design of the lower plenum and that the wedge could be removed from a redesign of the model geometry or that first jet could be plugged to eliminate the presence of the wall jet.

Figure 17 plots the time mean $X$-direction velocity $U$ for eight different profile locations: $x 1 \mathrm{u}, \mathrm{x} 1 \mathrm{~d}$, $x 3 u, x 3 d, x 4 u, x 4 d, x 6 u$, and x6d (see Figure 9). Several observations can be made regarding these results. First, it is striking how closely the trends for all three cases match each other. Second, the results for $U$ are much better at $x 6 \mathrm{u}$ and $\mathrm{x} 6 \mathrm{~d}$, downstream of the jet inlets, than for the region of the jets; the results for $U$ directly under Jet 2 at $x 1 \mathrm{u}$ and $\mathrm{x} 1 \mathrm{~d}$ show the greatest differences from the data. Third, the anomalous spikes that appear for the profiles at $\mathrm{x} 1 \mathrm{~d}, \mathrm{x} 3 \mathrm{u}$, and $\mathrm{x} 3 \mathrm{~d}$ are not exhibited by the CFD calculations, casting some doubt on whether they are truly present. The trends for $\mathrm{x} 4 \mathrm{u}$ and $\mathrm{x} 4 \mathrm{~d}$ are partially correct, partially incorrect. Overall, the agreement between the calculations for Cases 1-3 and the MIR data are not really satisfactory, though there are regions where it is fairly good, especially downstream of the jet region.

\subsubsection{Two-Equation Eddy-Viscosity Models}

Results have been obtained for three different two-equation, eddy-viscosity, turbulence models available in STARCCM+ (see Ref. 4). These are the standard $k \sim \varepsilon$ two layer model with the all $y^{+}$wall model, the AKN $k \sim \varepsilon$ low Re model with the all $y^{+}$wall model, and the Menter shear-stress transport $k \sim \omega$ model with the all $y^{+}$wall model. The latter two models are specifically designed to apply to within close proximity of the wall $\left(y^{+}<11\right)$, while the first model breaks the wall region into two layers: a viscous sublayer and a turbulent sublayer. However, the range of $y^{+}$puts the first nodes in the viscous sublayer. Turbulence inlet conditions are set to the default values of turbulence intensity $=0.01$ and turbulent viscosity ratio $=\mu_{t} / \mu=10$. Inlet mean vertical velocities are set to uniform values based on the bulk values measured from the mass flow rotameters. The initial conditions are set to mean velocities of $(1.0,0.0,0.0)$ $\mathrm{m} / \mathrm{sec}$. Each case is computed as a steady-state problem until the residuals do not reduce further. The calculations are then changed to unsteady. All of these cases maintain unsteady conditions throughout the rest of the computation time. The a 2 grid $(9.7 \mathrm{~mm}$ inlet height) is used for Cases $4-6$. Table 3 summarizes the two-equation, eddy-viscosity cases that have been computed.

Table 3. Summary of CFD model cases that employed two-equation, eddy viscosity models.

\begin{tabular}{|c|c|c|c|c|c|c|c|}
\hline Case & $\begin{array}{c}\text { Turbulence } \\
\text { Model }\end{array}$ & $\begin{array}{c}\text { Wall } \\
\text { Treatment }\end{array}$ & Grid & $\begin{array}{c}\text { Inlet } \\
\text { Velocity }\end{array}$ & $\begin{array}{c}\text { Inlet TI } \\
\text { (\%) }\end{array}$ & $\begin{array}{c}\text { Inlet Vis. } \\
\text { Ratio }\end{array}$ & $\begin{array}{c}\text { Simulation } \\
\text { Time (sec) }\end{array}$ \\
\hline 4 & $k \sim \varepsilon$ & all $y^{+}$ & $\mathrm{a} 2$ & uniform & 0.01 & 10 & 4.4 \\
\hline 5 & AKN & all $y^{+}$ & a2 & uniform & 0.01 & 10 & 2.8 \\
\hline 6 & $k \sim \omega$ & all $y^{+}$ & a2 & uniform & 0.01 & 10 & 3.2 \\
\hline
\end{tabular}


(a)
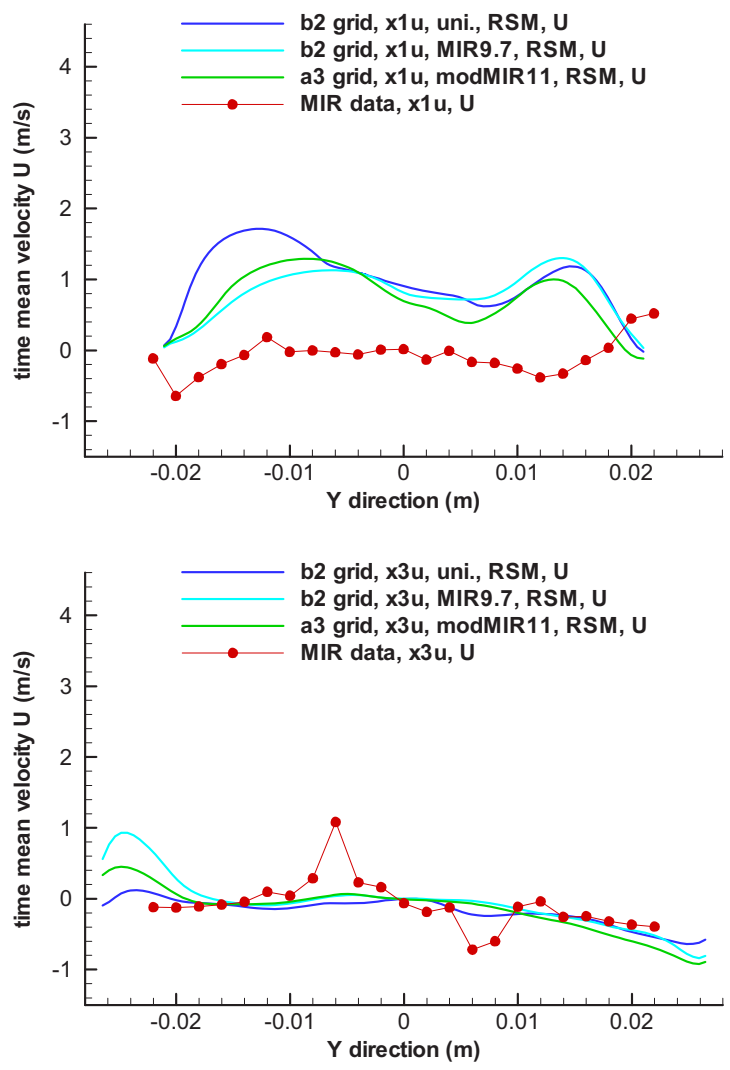

(c)

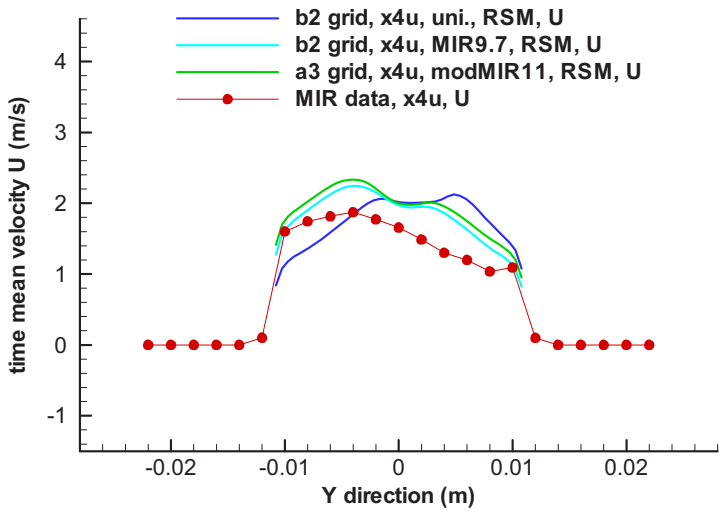

(e)

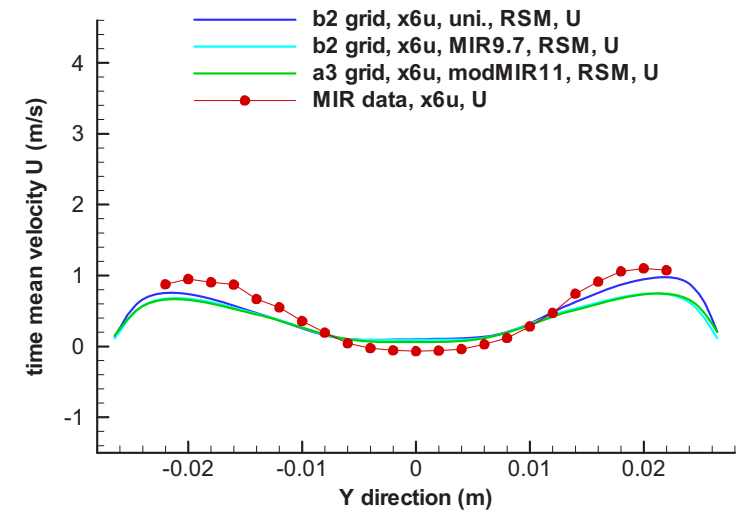

(g) (i)

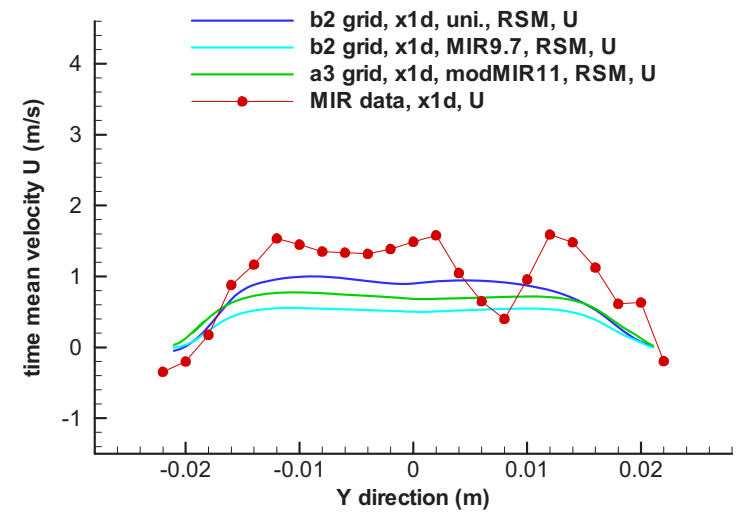

(d)

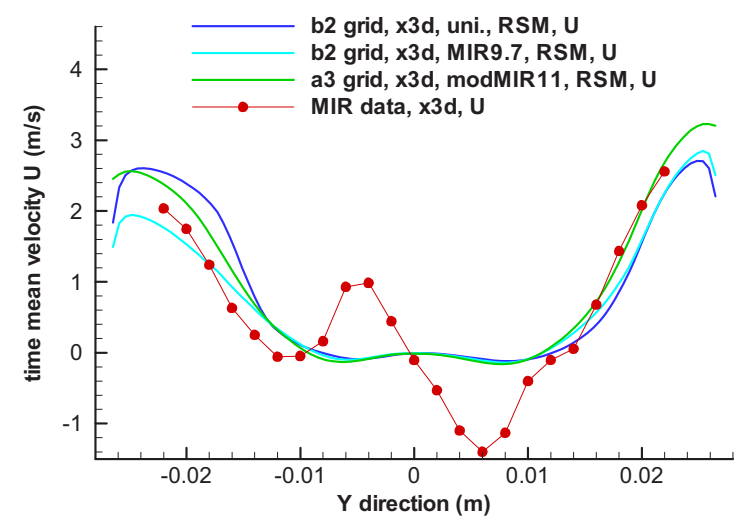

(f)
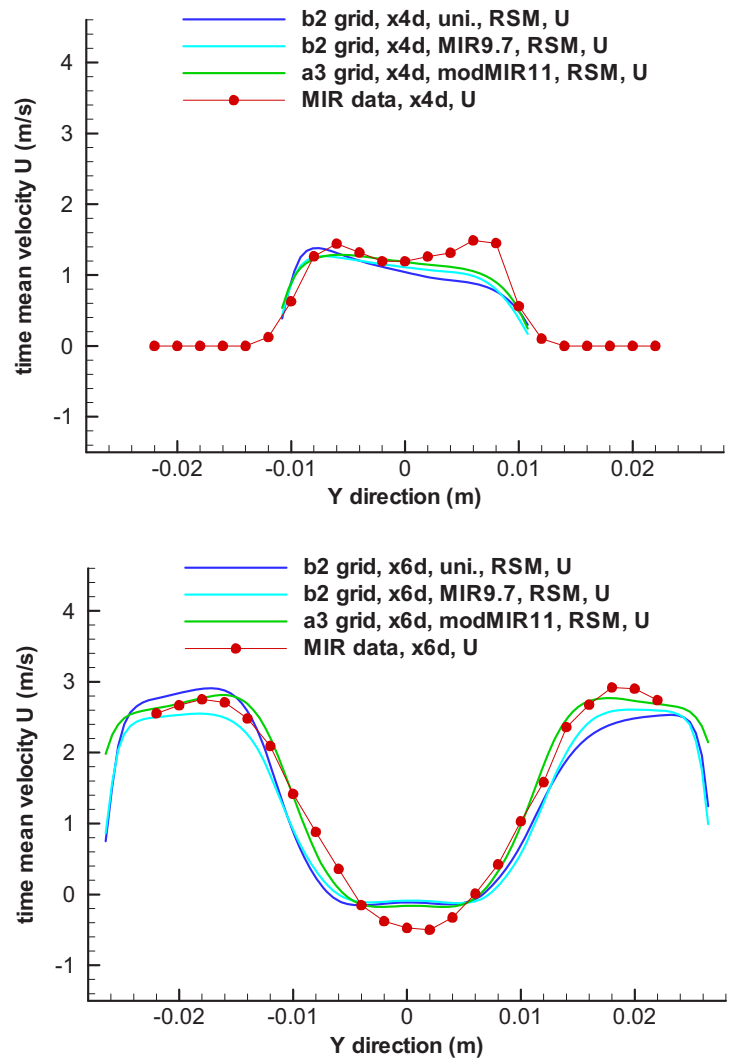

Figure 17. Profiles of time mean $U$ at $\mathrm{x} 1 \mathrm{u}, \mathrm{x} 1 \mathrm{~d}, \mathrm{x} 3 \mathrm{u}, \mathrm{x} 3 \mathrm{~d}, \mathrm{x} 4 \mathrm{u}, \mathrm{x} 4 \mathrm{~d}, \mathrm{x} 6 \mathrm{u}, \mathrm{x} 6 \mathrm{~d}$ for the three RSM cases. 
Figure 18 provides time traces of ensemble mean velocity $\langle u\rangle$ for the three eddy-viscosity cases at points $\mathrm{p} 5 \mathrm{~d}, \mathrm{p} 7 \mathrm{~d}$, and $\mathrm{p} 7 \mathrm{u}$. Unlike results shown above using the RSM, these results all indicate nonstationary flow. The standard $k \sim \varepsilon$ two layer traces are all very regular, while those for the other two models are very irregular, with oscillation amplitudes being greatest for the $k \sim \omega$ model and least for the standard $k \sim \varepsilon$ model. The irregularity of the oscillations in Figures $18 \mathrm{~b}$ and $\mathrm{c}$ is likely because of passing shed vortices from upstream, each with somewhat different shedding frequency. As shown in Figure 18, the typical vortex shedding time scale is $\geq 0.03 \mathrm{sec}$. The turbulent time scale for the large turbulent eddies is $\tau \sim 1 / \omega$ where $\omega$ is the specific dissipation of the $k \sim \omega$ model (see Ref. 5). For the present flow, the large turbulence time scale is of the order of $0.003 \mathrm{sec}$. This represents a separation of an order of magnitude between the turbulent and vortex-shedding time scales.

The anomalous spikes that can be seen for $\mathrm{p} 7 \mathrm{u}$ in Figure $18 \mathrm{~b}$ and for $\mathrm{p} 7 \mathrm{~d}$ in Figure 18c may simply be the infrequent coincidence of shed vortices that sum either amplitude peaks or troughs. The traces for point $\mathrm{p} 7 \mathrm{~d}$ exhibit the largest amplitude oscillations for all models. Additionally, the apparent long-time averages for point $\mathrm{p} 7 \mathrm{~d}$ are all rather different for the three models, while those for the other points are much closer. These results imply that there are some large variations in the flow dynamics in the flow near the inlet jets, causing the sometimes large variations in the point time signatures downstream.

The amplitude of the oscillations graphed in Figure 18 is indicative of the overall level of turbulent viscosity, that is, highest for the lowest amplitude oscillation. This would indicate that of the three turbulence models represented in Figure 18, the $k \sim \varepsilon$ model has the highest turbulent viscosity, the AKN model next and the $k \sim \omega$ model the lowest.

(a)

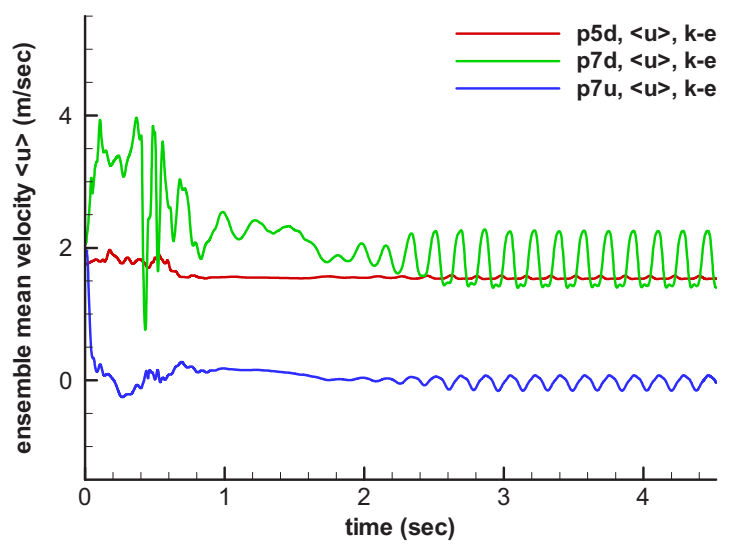

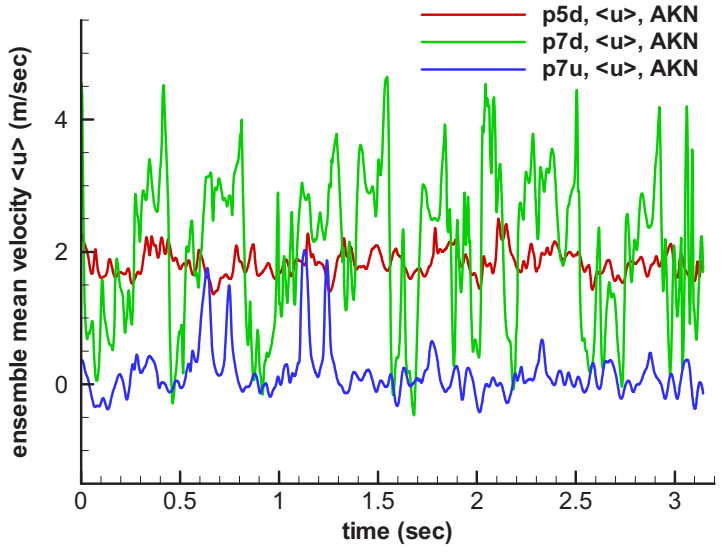

(b)

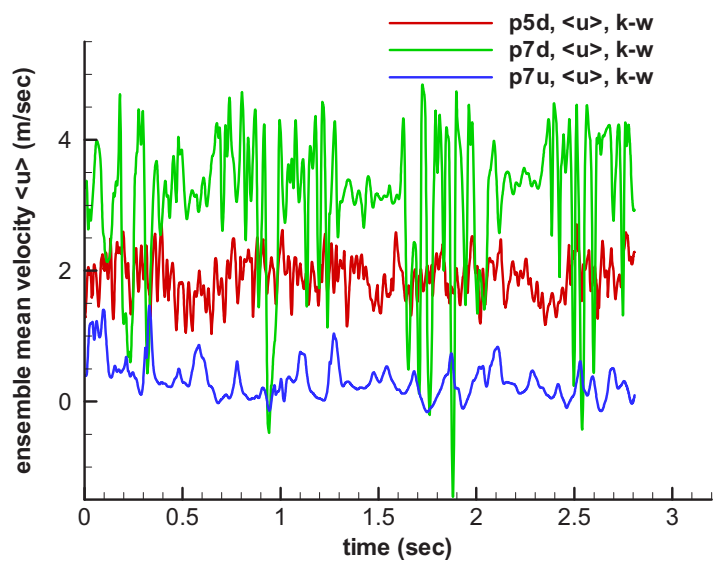

(c)

Figure 18. Time traces for ensemble mean velocity $\langle u\rangle$ at points $\mathrm{p} 5 \mathrm{~d}, \mathrm{p} 7 \mathrm{~d}$, and $\mathrm{p} 7 \mathrm{u}$ in the flow for the (a) standard $k \sim \varepsilon$ model, (b) AKN model, and (c) $k \sim \omega$ model. 
Figure 19 provides contour plots of the ratio of the turbulent viscosity to the constant molecular viscosity for the standard $k \sim \varepsilon$ two-layer model, AKN $k \sim \varepsilon$ model, SST $k \sim \omega$ model, and RSM (Case 3) at $\mathrm{Z}=-70 \mathrm{~mm}$. These figures show that in the region downstream of the jet inlets, where the time traces of Figure 18 are taken, the turbulent viscosity is indeed highest for the standard $k \sim \varepsilon$ model, lower for the AKN $k \sim \varepsilon$ model, and lower still for the $k \sim \omega$ model. The turbulent viscosity for the RSM appears to be higher than that for the $k \sim \omega$ model, but lower than that for the AKN model in the same region. In the region of the jet inlets, the RSM has the highest turbulent viscosity, the standard $k \sim \varepsilon$ model next, the $k \sim \omega$ model next and the AKN model the lowest.

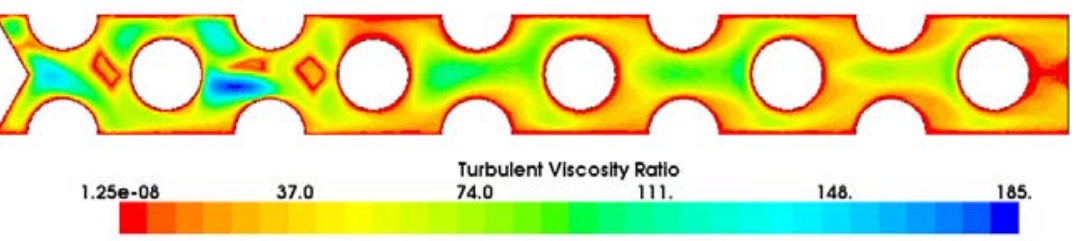

(a)

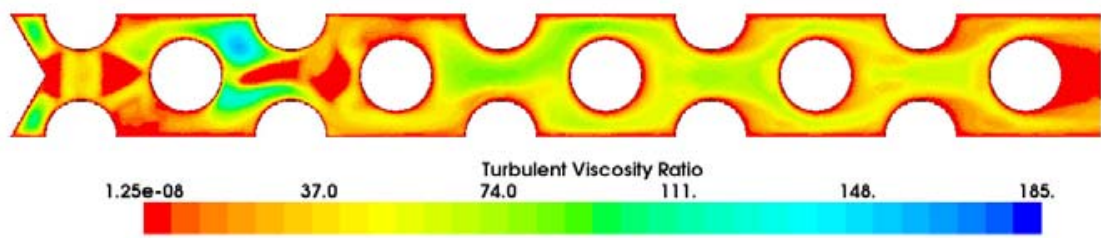

(b)

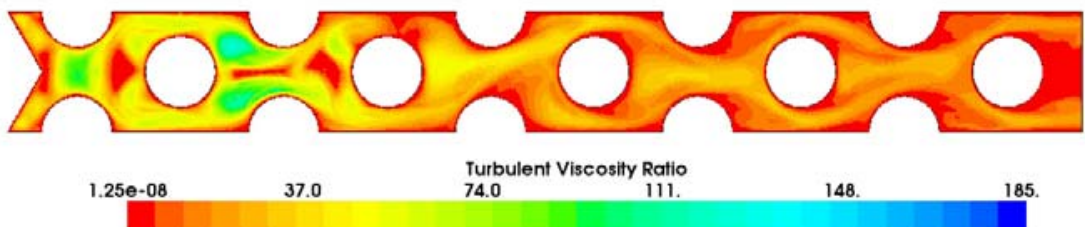

(c)

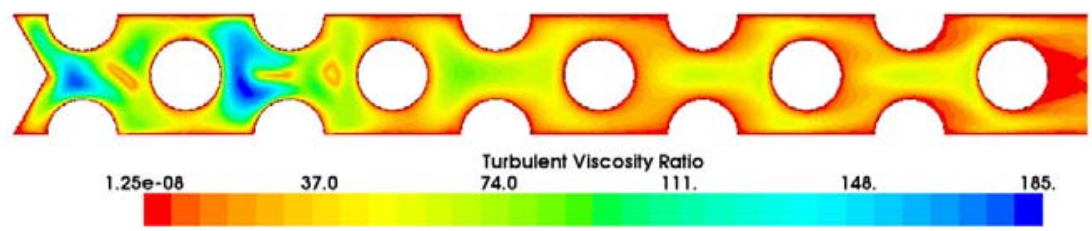

(d)

Figure 19. Ratios of turbulent to molecular viscosity for the (a) standard $k \sim \varepsilon$ model, (b) the AKN $k \sim \varepsilon$ model, (c) the $k \sim \omega$ model and the (d) RSM model (Case 3).

Figure 20 illustrates profiles for the time mean velocity $W$ at y0u and y0d locations for the three turbulence models. Velocities for the standard $k \sim \varepsilon$ model are averaged over four cycles because of the regularity of the cycles (Figure 18a). Velocities are averaged over the whole time for the other two models. At y $0 \mathrm{u}$, the signatures of the four jets are clearly visible in the MIR data. However, for the standard $k \sim \varepsilon$ model, the first jet is missing. Results for the other two models clearly show the first jet, albeit with lower magnitude than the data. These results are a clear improvement over the results shown earlier for the RSM cases, even for Case 1, Figure 15, which uses the rotameter inlet data. Hence, the location and strength of the first jet is significantly affected by both the inlet conditions and the turbulence model. The location of the second jet is also somewhat off for the three models, while the third and fourth jets are predicted in the correct locations, again with somewhat too low magnitude. 

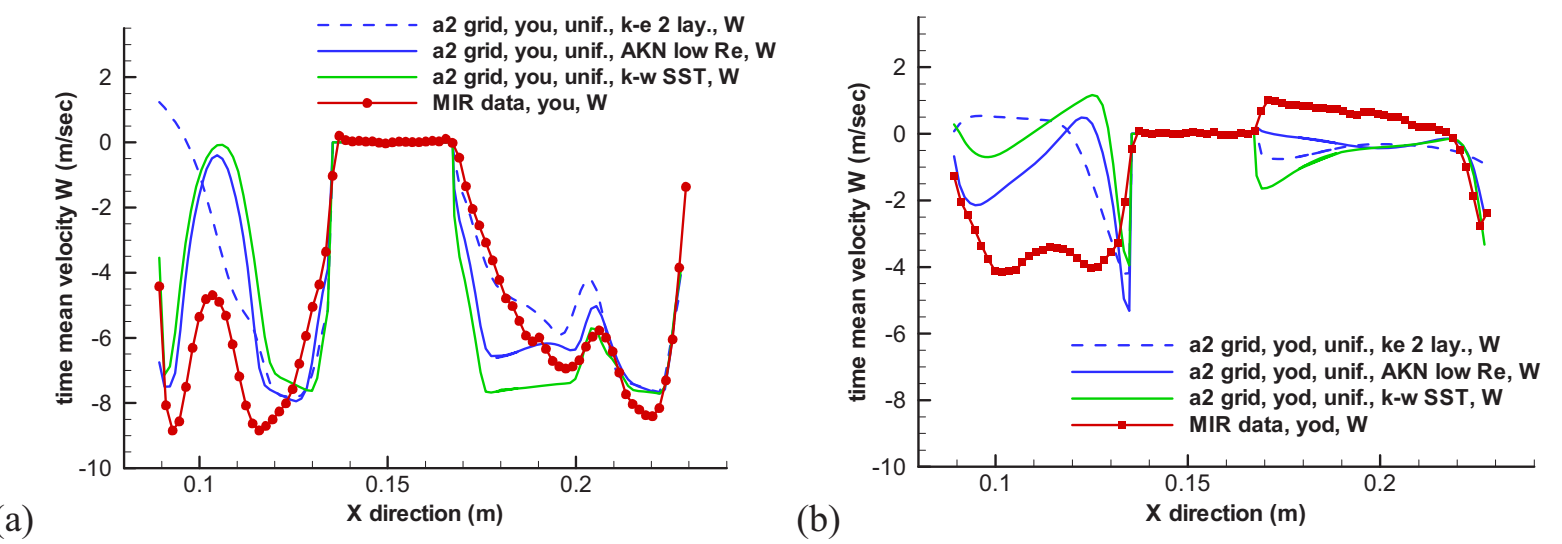

Figure 20. Time mean velocity $W$ at (a) y0u and (b) y0d for eddy-viscosity Cases 4-6 compared to the MIR data.

At y0d, the $W$ velocity profile is not well predicted by any of the models, though the predictions for the AKN $k \sim \varepsilon$ model are best. In particular, the signature for the first jet is too weak while that for the second jet is too strong; the locations for each are somewhat different from the data. The standard $k \sim \varepsilon$ and $k \sim \omega$ models exhibit a signature for the third jet when they shouldn't. In contrast, the results for the RSM cases, Figure 15b, show a better trend than for the eddy-viscosity models.

Figure 21 illustrates contour plots for the time mean velocity $W$ at $Z=-70 \mathrm{~mm}$ for the standard $k \sim \varepsilon$ model, the AKN $k \sim \varepsilon$ model, the $k \sim \omega$ model and the MIR data. (It should be noted that the MIR data do not extend to the outlet of the scaled model because of lack of optical access; also note that the contours levels are all matched.) This view clearly shows how the standard $k \sim \varepsilon$ model results are asymmetrically skewed to one side while those for the AKN $k \sim \varepsilon$ model, the $k \sim \omega$ model and the MIR data are close to symmetric. The fact that the latter two turbulence models are able to predict symmetry for the first two jets argues that the asymmetry is a result of the turbulence mode employed. The jet velocity magnitudes for Cases 4-6, however, are lower than for the MIR data. Interestingly, the shapes of the jet signatures are quite similar for the AKN model, the $k \sim \omega$ model results and the MIR data. Considering the six cases that have been computed, it is the case that the ones that were carefully designed to be valid in the viscous sublayer $\left(y^{+}<11\right)$ are the ones that show nearly symmetric results in these contour plots, that is the AKN model and the $k \sim \omega$ model.

It is interesting to note that the asymmetric results produced using the RSM and standard $k \sim \varepsilon$ models all show similar time traces for the several points shown in Figures 14abc and 18a, that is, either going to steady state or oscillating with a low amplitude, relatively long-time wavelength, $\geq 0.1 \mathrm{sec}$., a wavelength that is longer than that of the typical shedding vortices seen in Figures $18 \mathrm{bc}(\sim 0.03 \mathrm{sec}$.). These cases may have found stable or semistable asymmetric solutions that are a function of the turbulence model or the wall treatment or both. Because the asymmetry mainly occurs in the region near the first inlet jet and wedge, it is possible that the presence of the wedge is what is creating the asymmetric semistable solutions. If this is the case, it would be better to eliminate the wedge to avoid this situation. That is, it is not desirable to have a validation data set that is based on flow in a geometry that displays semistable solutions, which may be a result of the flow rate or the nature of the geometry or both. 


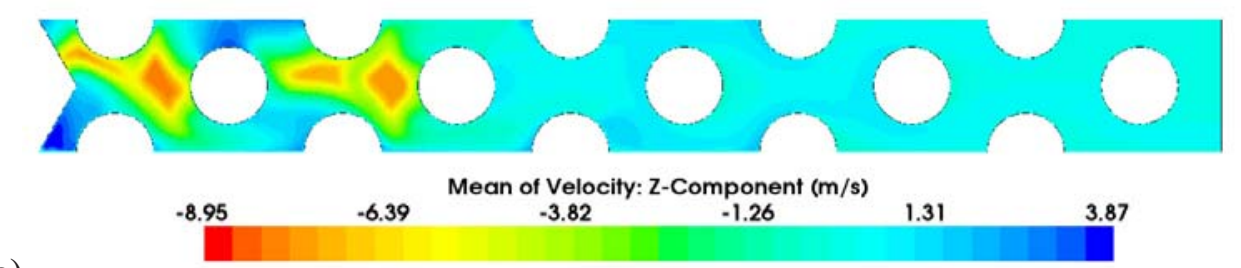

(a)

(b)
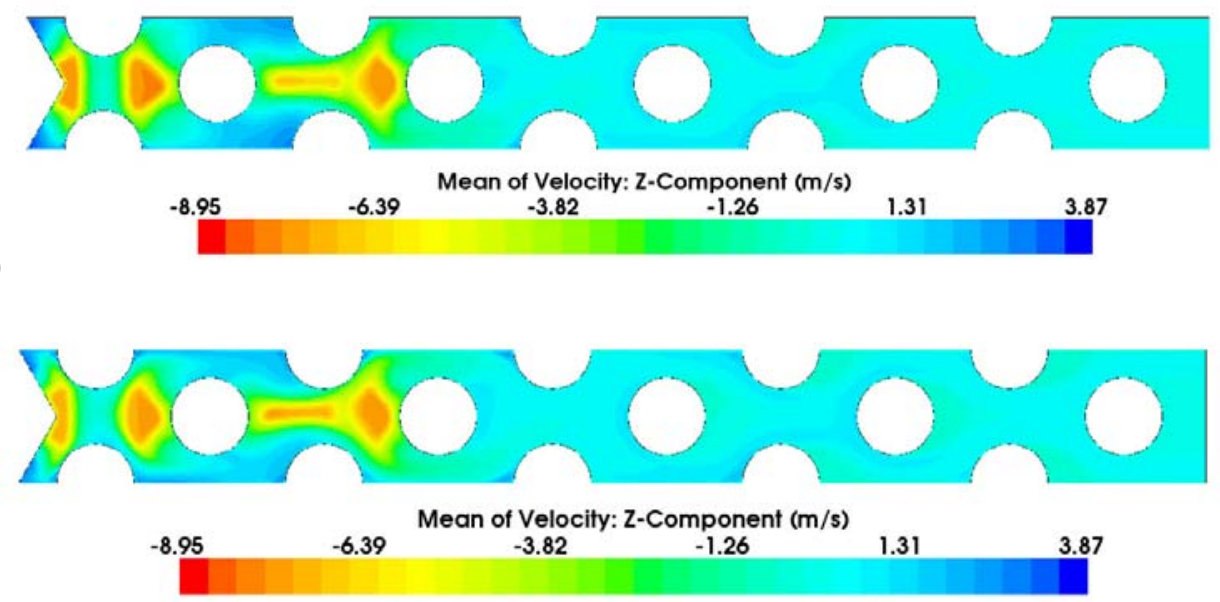

(c)

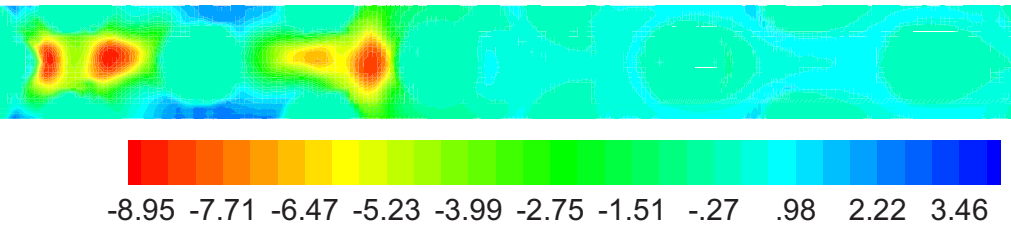

Figure 21. Contour plots for time mean velocity $W$ at $\mathrm{Z}=-70 \mathrm{~mm}$ for the (a) standard $k \sim \varepsilon$, (b) AKN, (c) $k \sim \omega$ eddy-viscosity models, and the (d) MIR data.

Figure 22 plots the time mean $\mathrm{X}$-direction velocity $U$ for the eight different profile locations, $\mathrm{x} 1 \mathrm{u}$, $\mathrm{x} 1 \mathrm{~d}, \mathrm{x} 3 \mathrm{u}, \mathrm{x} 3 \mathrm{~d}, \mathrm{x} 4 \mathrm{u}, \mathrm{x} 4 \mathrm{~d}, \mathrm{x} 6 \mathrm{u}$, and x6d (see Figure 9) for the standard $k \sim \varepsilon$ model, AKN model, and $k \sim \omega$ model. Profiles for the three turbulence models are quite different from each other and from the data for profiles $x 1 \mathrm{u}$ and $\mathrm{x} 1 \mathrm{~d}$, located below the second inlet jet. The AKN results are quite symmetric, while the $k \sim \omega$ results are somewhat asymmetric and the $k \sim \varepsilon$ results are very asymmetric, as are the MIR data though not in the same way. Results for the three cases at $x 3 \mathrm{u}$ and $\mathrm{x} 3 \mathrm{~d}$ are rather different from each other and show poorer quantitative and qualitative agreement with the data than the three RSM cases (see Figure 17). Again, the anomalous spikes/bumps exhibited by the MIR data for these profiles are mostly missed by the calculations. The calculations for locations $\mathrm{x} 4 \mathrm{u}$ and $\mathrm{x} 4 \mathrm{~d}$ show better agreement with the MIR data, except for the standard $k \sim \varepsilon$ model at $\mathrm{x} 4 \mathrm{~d}$, where the trend is dramatically wrong. Beyond the inlet jet region, calculations at $\mathrm{x} 6 \mathrm{u}$ and $\mathrm{x} 6 \mathrm{~d}$ show quite reasonable agreement, as did the RSM cases above, especially for the AKN model, which agree quite well with the data. The agreement for the AKN model with the MIR data for both x6 profiles is better than for the RSM. However, overall, the agreement between the three eddy-viscosity models is not satisfactory for either the $U$ or the $W$ velocity components, though there are regions of agreement, especially downstream from the jet inlet region. 
(a)

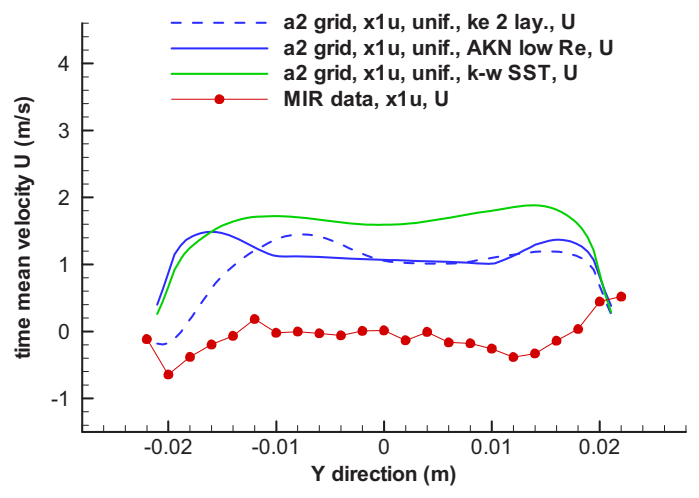

(c)
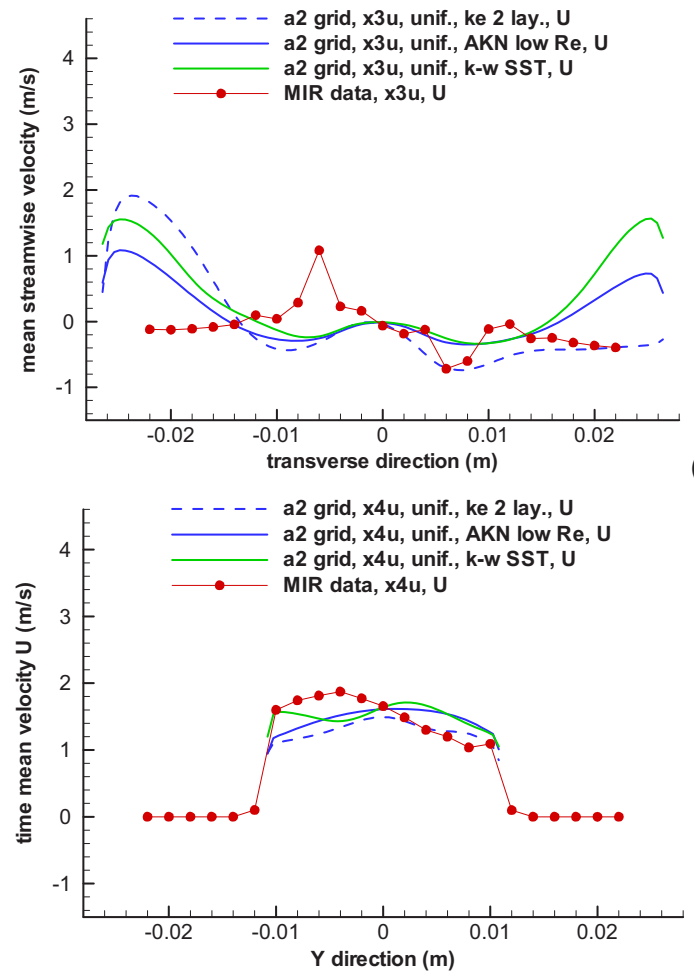

(e) (b)

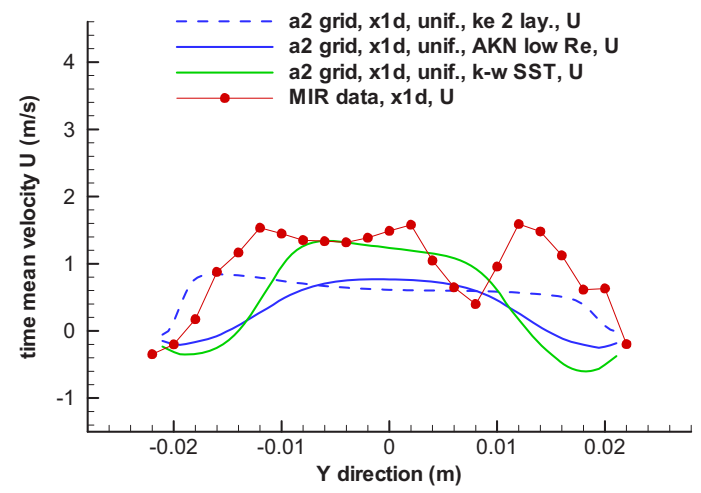

(d)
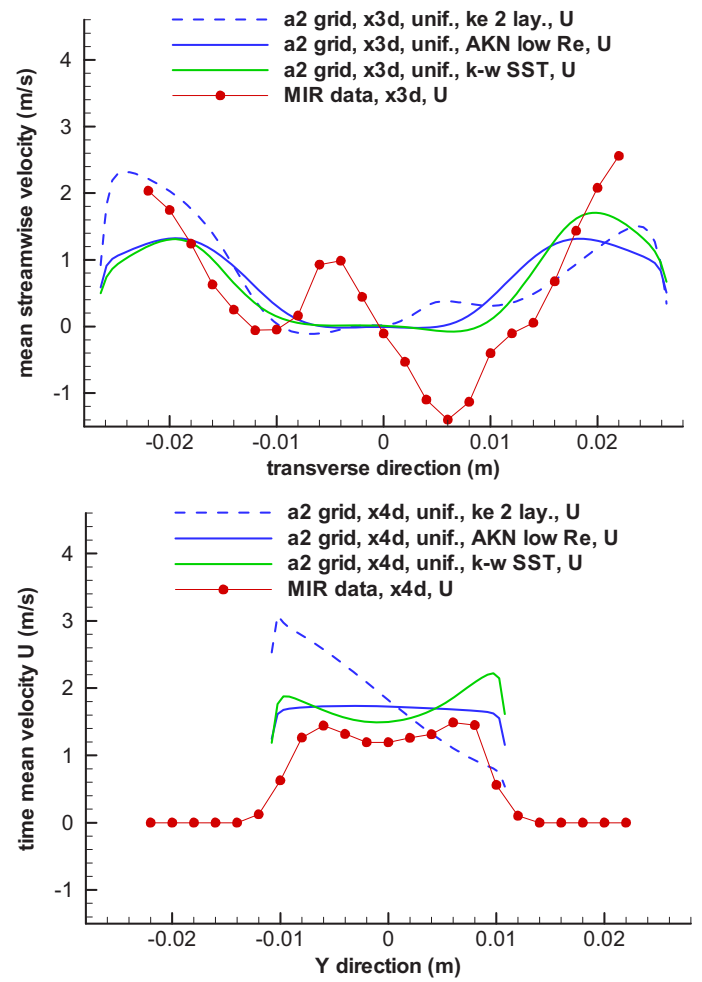

(f)
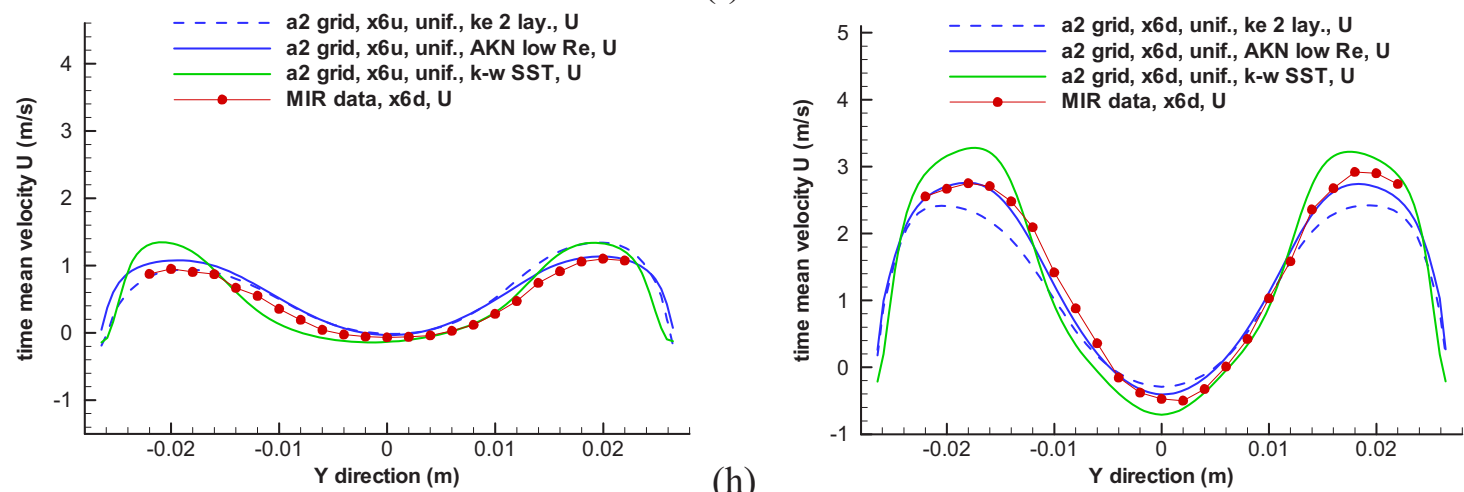

(h)

Figure 22. Profiles of time mean $U$ at $x 1 u, x 1 d, x 3 u, x 3 d, x 4 u, x 4 d, x 6 u, x 6 d$ for the 3 eddy-viscosity cases. 


\section{SUMMARY AND RECOMMENDATIONS}

Experimental data were previously taken in the INL's MIR test facility for a scaled section of the lower plenum of the VHTR reactor to provide a CFD validation data set for CFD calculations to be used in designing and analyzing the VHTR. This report presents results of CFD simulations of the flow in the scaled model performed to investigate the suitability of the data to become a validation data set or standard problem. Recommendations based on several of the findings obtained from the CFD analyses of the flow are discussed in this section.

Computations for several variations of initial conditions, inlet conditions, and turbulence models have been obtained for the flow geometry. Initial conditions have been prescribed in two different ways. For the first method, steady state computations are made until convergence slows or stops, usually not to a converged state, after which unsteady calculations are begun. The second method simply starts from stagnant conditions. Overall, the variation in initial conditions had little effect.

The inlet conditions used come from two different sources. The first source is the mass flow rotameters that were connected inline with each of the four inlet jets, and used to adjust the mass flows for the experiment. The second source is the PIV data that were taken in the inlet jet ducts above the inlet plane. The inlet conditions based on the rotameters data only provide a uniform velocity for the component normal to the inlet duct cross-sections. The inlet conditions based on PIV data taken in the duct inlets contain detailed data for the three velocity components and turbulent kinetic energy. PIV data taken at two locations, 9.7 and $11 \mathrm{~mm}$ above the inlet plane, have been used. Flow rates computed by integrating the PIV data fall below the rates provided by the rotameters, especially for the first jet. The $9.7 \mathrm{~mm}$ data, for which the total mass flow rate is $10.4 \%$ low, are used as is, while the vertical velocity component for the $11 \mathrm{~mm}$ data, for which the total flow rate is $6.7 \%$ low, is increased uniformly to match the total flow rate of the rotameter data. The results for the computations using the inlet conditions based on the rotameters show significant variations from the results using the MIR inlet data, especially in the location of the first inlet jet, even using the same turbulence model. This result is partly attributed to the fact that the mass flows for the PIV data are too low. This discrepancy is a serious shortcoming of the MIR data and should be remedied. It is recommended that additional MIR data be taken and that the inlet ducts be measured with laser sheets that illuminate the duct cross-sections such that dense inlet data can be obtained that should provide close agreement with the mass flow rotameters.

Four turbulence models (in the STARCCM+ commercial CFD code) are used to simulate the MIR data: standard $k \sim \varepsilon$ two-layer model, AKN low Re $k \sim \varepsilon$ model, and Menter SST $k \sim \omega$ model, all with all $y^{+}$ wall treatments, and RSM with linear pressure strain and high $y^{+}$wall treatment. Results for the several models show various overall levels of turbulent viscosity, very different dynamic behavior in the flow patterns, variations in asymmetry, and variations in predicted velocity as follows:

- The standard $k \sim \varepsilon$ model and the RSM showed the greatest turbulent viscosity, the $k \sim \omega$ model the least, and the AKN model somewhat more than the $k \sim \omega$ model.

- The RSM results appeared to be diminishing in their unsteadiness toward a steady state condition, which is inconsistent with vortex-shedding.

- The greatest departure of the several cases from the MIR data is in the vicinity of the first jet. This may be because of the turbulence model and/or the wall treatment or it may be because of the presence of the wedge. The presence of the wedge causes the first jet to be a wall-jet. The ability of the turbulence models to accurately represent a wall jet should perhaps be examined in a separate effects problem if wall jets are, in fact, important to the flow in the lower plenum; this may not be the case because the final design has not yet been determined.

- Significant asymmetry is seen in the results for the standard $k \sim \varepsilon$ and RSM models while the $k \sim \omega$ and AKN model results are generally quite symmetric. 
The issue, how dynamic is the actual flow, should be resolved by experiment. However, the MIR data were taken at two-three frames per second, not nearly fast enough to even track vortex shedding. It is recommended to obtain data using a faster system to see how dynamic the actual flow really is, which will help sort out the turbulence modeling issue. It will also help to answer the question: Are multiple unstable flow patterns present that are complicating the ability of CFD modeling to capture them? This will have ramifications regarding turbulent heat transfer, which is directly related to the primary concerns regarding the hot helium flow in the lower plenum. That is: How hot are local hot spots and how good is the thermal mixing of the helium in the lower plenum?

Finally, it is clear from the above CFD investigation that CFD should be used in the design of new standard problems. The application of CFD will help the experiment to be designed to have inlet and outlet boundaries that can be properly simulated and will help in characterizing the flow itself to know what measurements are best, at which locations, using which data acquisition techniques. CFD will also help suggest which phenomena should be examined separately in simpler standard problems, primarily to see how well various turbulence models perform.

\section{REFERENCES}

1. H. M. McIlroy, D. M. McEligot, and R. J. Pink, "Measurement of Flow Phenomena in a Lower Plenum Model of a Prismatic Gas-Cooled Reactor," Proceedings of the $16^{\text {th }}$ International Conference on Nuclear Engineering (ICONE-16), Orlando, FL, May 11-15, 2008.

2. R. W. Johnson, "Modeling Strategies for Unsteady Turbulent Flows in the Lower Plenum of the VHTR," Nuclear Engineering \& Design, 238, pp. 482-491, 2008.

3. R. W. Johnson, "Development of a CFD Analysis Plan for the First VHTR Standard Problem," Proceedings of the $4^{\text {th }}$ International Topical Meeting on High Temperature Reactor Technology (HTR2008), paper HTR2008-5828, Washington, D. C., Sept. 28 - Oct. 1, 2008.

4. FLUENT, Version 6.3.26, 2008, FLUENT Inc., 10 Cavendish Court, Centerra Resource Park, Lebanon, NH, 03766.

5. STAR-CCM+, Version 3.06, 2008, CD-adapco, 60 Broadhollow Road, Melville, NY 11747.

6. R. W. Johnson, "CFD Investigation Of Experimental Data Proposed To Be A Validation Data Set," Proceedings of the 17th International Conference on Nuclear Engineering (ICONE17), paper ICONE17-75604, Brussels, Belgium, July 12-16, 2009.

7. R. W. Johnson, "Examination of a Proposed Data Set Using CFD Calculations," Proceedings of the ASME 2009 Fluids Engineering Summer Meeting (FEDSM2009), paper FEDSM2009-78317, Vail, Colorado, August 2-5, 2009. 
Appendix A

Standard Model Problem Geometry 


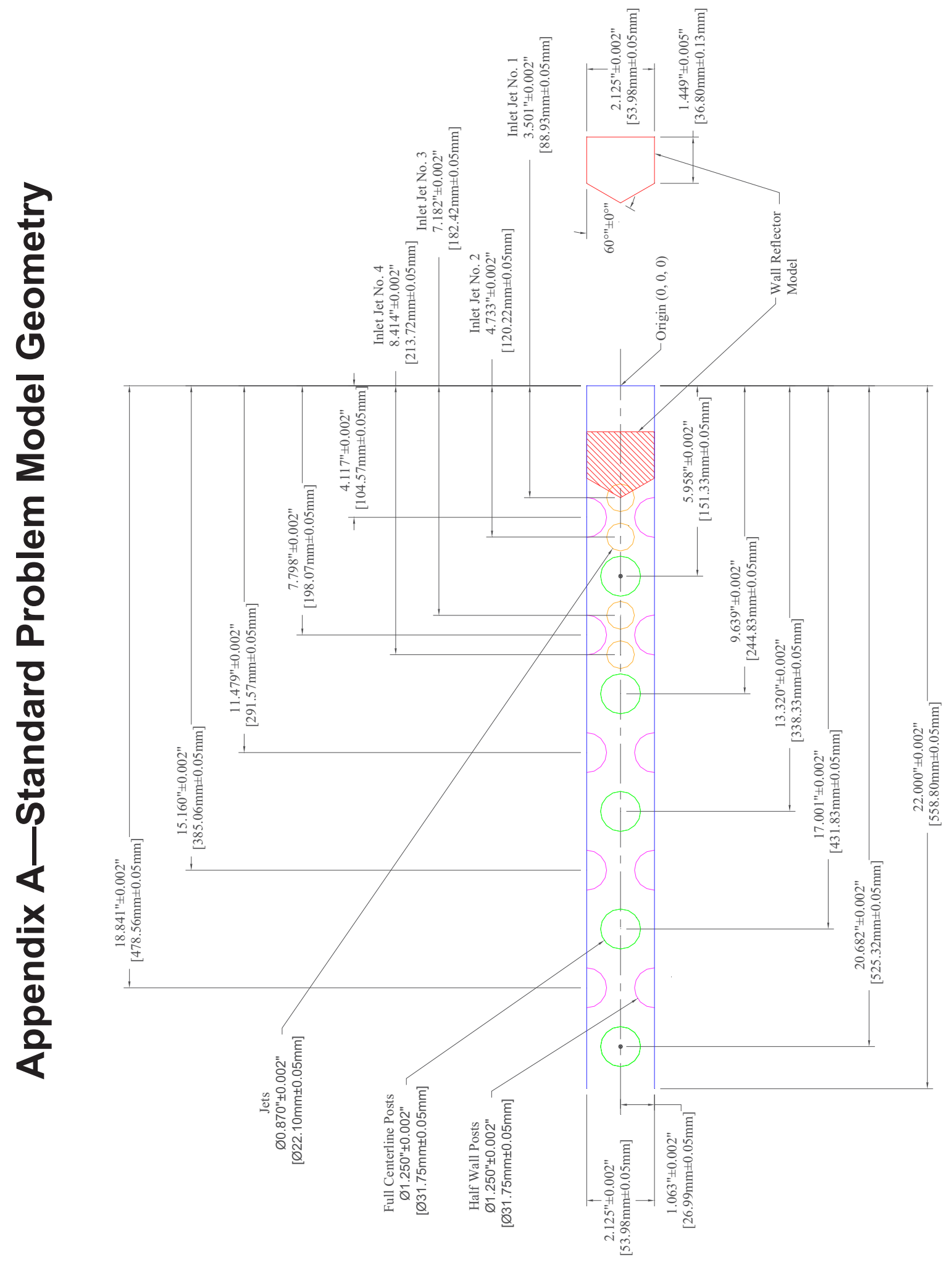

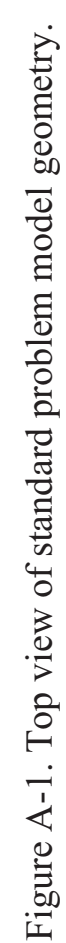




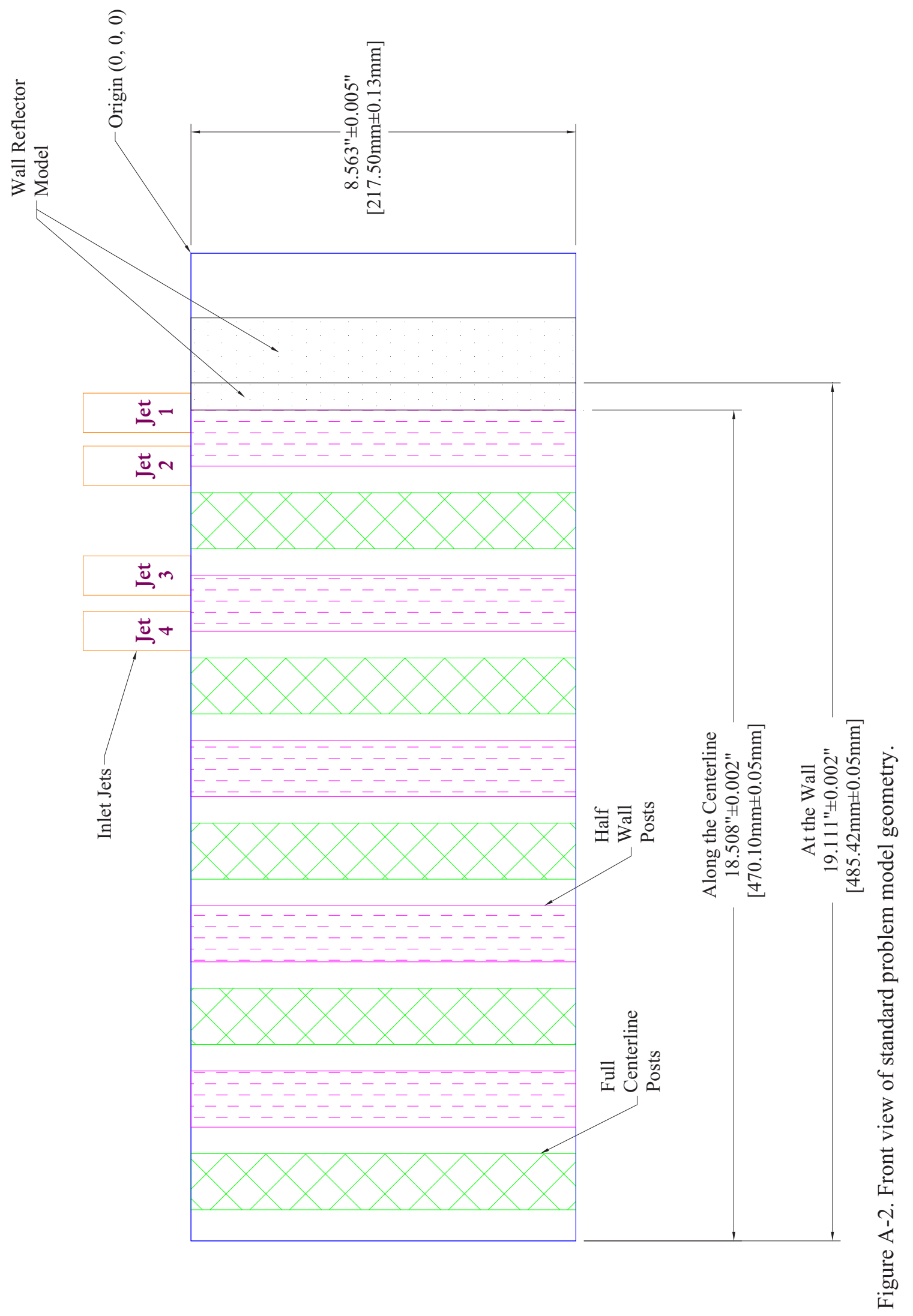

